\title{
Particle-Vortex Duality and the Modular Group: Applications to the Quantum Hall Effect and Other 2-D Systems
}

\author{
C.P. Burgess ${ }^{1,2}$ and Brian P. Dolan ${ }^{3}$ \\ ${ }^{1}$ Institute for Advanced Study, Princeton, NJ, 08540. \\ ${ }^{2}$ Physics Department, McGill University, 3600 University Street, Montréal, Québec, Canada H3A 2T8. \\ ${ }^{3}$ Dept. of Mathematical Physics, National University of Ireland, Maynooth, Republic of Ireland. \\ e-mail: cliff@physics.mcgill.ca, bdolan@thphys.may.ie
}

(October 2000)

\begin{abstract}
We show how particle-vortex duality implies the existence of a large non-abelian discrete symmetry group which relates the electromagnetic response for dual two-dimensional systems in a magnetic field. For conductors with charge carriers satisfying Fermi statistics (or those related to fermions by the action of the group), the resulting group is known to imply many, if not all, of the remarkable features of Quantum Hall systems. For conductors with boson charge carriers (modulo group transformations) a different group is predicted, implying equally striking implications for the conductivities of these systems, including a super-universality of the critical exponents for conductor/insulator and superconductor/insulator transitions in two dimensions and a hierarchical structure, analogous to that of the quantum Hall effect but different in its details. Our derivation shows how this symmetry emerges at low energies, depending only weakly on the details of dynamics of the underlying systems.
\end{abstract}

PACS nos: 73.40.Hm, 05.30.Fk, 02.20.-a

\section{INTRODUCTION}

Two-dimensional electron systems have remarkable properties, including the quantum Hall effect and metalinsulator transitions, many features of which still resist theoretical explanation. The difficult part of describing these systems is that they involve strong correlations, and no small parameters present themselves to help with the analysis. This deprives theorists of most of the tools in their conceptual toolboxes.

Two kinds of theoretical tools which have proven useful for analysing these kinds of strongly-coupled problems are the exploitation of symmetries and of the simplifications which are associated with the low-energy limit. Duality symmetries, in particular, are likely to be useful since these typically relate strongly-coupled degrees of freedom to weakly-coupled ones, and in two dimensions particles and vortices make natural candidates for dual partners. Indeed, particle-vortex duality has been used several times in the literature to describe some aspects of both the Quantum Hall effect and conductor-insulator transitions in superconducting films.

Duality symmetries are unusual in that they are not symmetries in the usual sense that they need commute with the system's Hamiltonian. Instead, they relate two different kinds of systems to one another. This relation-

*Systems for which dualities commute with the Hamiltonian ship is useful when one of the two systems so related can be analysed, permitting conclusions to be drawn for its dual by acting with the duality transformation. Alternatively, duality can be useful if it maps a family of systems into themselves, since duality invariance then constrains how one flows amongst members of the family as external parameters - like temperature or magnetic field - are varied.

Our aim is to show that both of these lines of argument have very general applications to two-dimensional systems. In particular, our main result is to show that the twin operations of particle-vortex duality [1, 1 and the addition of $2 \pi$ units of statistics to charge carriers (which does not change their statistics at all) take a very simple form when expressed in terms of the material's electromagnetic (EM) response functions. These relations hold for any system for which the low-energy EM response is dominated by the motion of quasi-particles or vortices, and for which the dynamics of these quasiparticles and vortices are similar (in a way we make more explicit in what follows). Since these two transformations do not commute, they generate an infinite discrete group of duality relations amongst the EM response functions, and it is this large group which underlies the predictions

\footnotetext{
make up the special case of self-dual systems.

${ }^{\dagger}$ See references [5 80 for other approaches to duality in Quantum Hall systems.
} 
which we shall find.

In general the duality transformations we find do not preserve the momentum dependence of the EM response function and so, for instance, can relate materials whose response is very different (such as by relating superconductors to insulators). It turns out that conductors are mapped into themselves, however. When specialized to conductors, with the electromagnetic response characterized by the Ohmic and Hall conductivities, $\sigma_{x x}$ and $\sigma_{x y}$, the action of duality takes the form of subgroups of the modular group, $P S L(2, Z)$, acting on the complex conductivity, $\sigma=\sigma_{x y}+i \sigma_{x x}$ 用 as follows:

$$
\tilde{\sigma}=\frac{a \sigma+b}{c \sigma+d}
$$

with the integers $a$ through $d$ satisfying $a d-b c=1$ (the occurrence of this symmetry in a statistical mechanical model was first noticed in [9], in an investigation aimed at understanding QCD). The duality transformations as defined in eq. (11) are not symmetries of the Hamiltonian since, for instance, dual pairs differ in their electromagnetic response. Rather, these transformations are symmetries of the flow obtained as external parameters, such as magnetic fields and temperatures, are varied.

Which particular subgroup is important depends on the statistics of the charge carrying quasi-particles of the problem. If they are fermions, or related to them by the symmetries we shall describe, then the subgroup (denoted $\Gamma_{0}(2)$ in the mathematics literature [10]) is defined by the condition that the parameter $c$ must be even. For bose charge carriers, or their symmetry partners, such as for superconducting films or Josephson Junction arrays, the symmetry is instead $\Gamma_{\theta}(2)$, defined by the condition that $a, d$ are both odd and $b, c$ are both even, or vice versa. For both cases powerful predictions follow from the fact that our derivation shows that the symmetry constrains how the conductivities change as external variables are varied.

For Quantum Hall systems, the group $\Gamma_{0}(2)$ has been long conjectured to be important [11] 13 , and has been derived for these systems within a mean-field approximation [12]. For these systems our derivation accomplishes two new things. First, although our arguments are modelled on those of ref. [12], ours have a broader domain of validity since they explicitly assume only that quasiparticles or vortices dominate the low-energy EM response, and that the systems are clean enough to exclude any interactions which might distinguish quasi-particles from vortices, and so thereby ruin the duality symmetry which relates them. Because the fields we treat only arise in the effective theory, and are not meant to describe the

\footnotetext{
${ }^{\ddagger}$ We use units for which $e^{2} / h=1$.
}

complete electron dynamics, the mean-field approximation is kept under better control.

Second, our derivation helps clarify the assumptions which underlie analyses of the consequences of $\Gamma_{0}(2)$ invariance for the renormalization-group (RG) flow in the $\sigma_{x x}-\sigma_{x y}$ plane, since we show that this only relies on the underlying particle-vortex duality and on the long-wavelength limit. This is important because it has been shown [13 15] that most of the unique features of Quantum Hall electromagnetic response follow from the consistency of $\Gamma_{0}(2)$ invariance with RG flow in the $\sigma_{x x}-\sigma_{x y}$ plane, independent of the detailed form of the flow's $\beta$-function. (The constraints on $\beta$ which follow from this symmetry have also been considerably explored 14,16 19 .) Previously the key assumption of twodimensional flow, governed by $\Gamma_{0}(2)$ invariance, was just that: an assumption, although a plausible one motivated by analogy with the two-dimensional scaling theory of disorder [20 23]. In particular, since the scaling theory strictly only applies near the system's critical points the scaling motivation could not explain why many of the predictions following from $\Gamma_{0}(2)$-invariant flow work extremely well, even away from the flow's critical points. Since the derivation presented here is not similarly restricted to scaling regions, it explains why these otherwise surprisingly successful predictions work.

The identification of $\Gamma_{\theta}(2)$ as the duality symmetry for two-dimensional conductors with bosonic charge carriers is new, although some precursors of this idea exist. Implications of the particle-vortex generator of the group for critical behaviour in a superconductor-insulator transition have been examined [1], and the group $\Gamma_{\theta}(2)$ was discussed as potentially playing a role in two-dimensional systems 11, 13], including possibly for the Quantum Hall effect [5]. The action of $\Gamma_{\theta}(2)$ on longitudinal conductivities was written down in [24], although this was not extended to the whole upper-half complex plane.

Also new to this paper are the detailed predictions which follow for bosonic systems from the proposed symmetry group, and which are direct analogs of the symmetry consequences which are already known for Quantum Hall systems.

To which real-life systems does our duality symmetry apply? Just as it is difficult to compute ab initio whether a material will be a solid or not, it is difficult to answer from first principles which systems must be particlevortex symmetric. There are several things which can be said, however. First, if the symmetry holds, then all of its consequences follow together. For example, for Quantum Hall systems the semi-circle law, $\rho_{x x} \rightarrow 1 / \rho_{x x}$ duality, super-universality of the critical exponents, universality of the transition conductivities, odd-denominator fractional quantization of $\sigma_{x y}$ when $\sigma_{x x}=0$, the selection rules for which plateaux may be related by transitions, etc. must all come together as a package.

Second, since particle-vortex duality relies on the 
equivalence of the kinematics and couplings of the chargecarrying quasi-particles and vortices, it should be a good approximation when the only quantities of interest in the Hamiltonian are those describing the kinematics of these particles, and their couplings to the fields which describe the long-range vortex interactions and the electromagnetic fields which are applied to test the EM response. Duality could be ruined by other microscopic interactions which treat quasi-particles and vortices differently, such as from couplings with disorder or with other electronic degrees of freedom. Of course, disorder can also play other spoiling roles, such as by destroying the phase coherence on which the quantum regime which we assume depends.

A sufficient condition for particle-vortex duality, and its associated non-abelian extensions, might therefore be that the system be sufficiently clean to justify the neglect of other particle and vortex interactions when calculating the electromagnetic response. Although this condition is not strictly necessary, since our derivation also applies in the presence of any particle/vortex-democratic interactions, weak coupling is also implicit in our neglect of anomalous dimensions when deciding the relevance or irrelevance of low-energy interactions. Notice that it is the weakness of the quasi-particle couplings which are important in this decision, and the assumption that these are weak does not imply that the underlying electrons must also be weakly coupled in the microscopic Hamiltonian.

Now to the main arguments. We organize our presentation as follows: First we describe the action describing the low-energy dynamics of a system of quasi-particles and vortices, and cast it into a form which emphasizes the similarities between these two kinds of charge carriers. Next, we derive the action of the two basic symmetries $-2 \pi$ statistics addition and particle-vortex duality - for the electromagnetic response functions. Then, we specialize the result to the particularly interesting case of a conductor, to derive the action of the symmetry on the conductivities, $\sigma_{x x}$ and $\sigma_{x y}$. We briefly review the Quantum Hall case, where the charge carriers are fermions, and then repeat the analysis for charge carriers satisfying bose statistics, listing many experimental predictions which follow from the symmetries.

\section{PARTICLES AND VORTICES}

This section has two goals. First, we derive an expression for the effective action governing the low-energy interactions of charged quasi-particles and vortices for which the duality between these two kinds of objects is made explicit. Second, we compute the EM response for a system of such charges and vortices, for use when deriving the implications of particle-vortex duality in the next section.

\section{A. The Effective Quasi-particle-Vortex Action}

Our starting point is the following lagrangian, which describes the low-energy/long-wavelength interactions of a collection of $N_{p}$ quasi-particles and $N_{v}$ vortices with a weak electromagnetic probe, $A_{\mu}$ :

$$
\begin{aligned}
\mathcal{L}_{\mathrm{eff}}= & -\frac{\pi}{2 \theta} \epsilon^{\mu \lambda \nu} a_{\mu} \partial_{\lambda} a_{\nu}+\mathcal{L}_{p}(\xi, a+A) \\
& -\frac{\kappa}{2}\left[\partial_{\mu} \phi-q_{\phi}\left(a_{\mu}+A_{\mu}\right)\right]\left[\partial^{\mu} \phi-q_{\phi}\left(a^{\mu}+A^{\mu}\right)\right] \\
& +\cdots,
\end{aligned}
$$

which we write in the continuum approximation because our interest is directed towards the low-frequency, longwavelength EM response.

Several features of this lagrangian bear explanation, and since our final results ultimately depend on its validity, we pause here to explain its form in some detail.

- Why Both Particles and Vortices?: Usually charged particles and vortices, in the way we define them, do not coexist in the low-energy theory, since the vortices presuppose the breaking of electromagnetic symmetries which precludes the existence of isolated electrically charged particles, and our later applications only require the consideration of systems containing one or the other. We nonetheless use the mathematical device of keeping both in eq. (2) since it permits us to derive our results for particles only and vortices only by taking the appropriate limits of a single formula.

- Field Content: $A_{\mu}$ is the small external electromagnetic potential which is applied to probe the system's EM response, $\xi_{k}^{\mu}(t)$ is the position of the $k$ 'th quasi-particle of the system as a function of time, and $a_{\mu}$ is the usual statistical gauge potential which ensures that the interchange of two quasi-particles produces the phase $e^{i \theta}$ [25]. $(\theta=2 \pi n$, for integer $n$, corresponds to bosonic quasiparticles, while $\theta=(2 n+1) \pi$ describes fermionic quasiparticles.) 3

- Relativistic Form: We use relativistic notation in eq. (2) in order to most cleanly illustrate the logic of the argument. This proves to be convenient because the relativistic case shows all of the main features of duality, and is considerably simpler to describe. We have checked that other features of a non-relativistic treatment - such as the potential appearance of kinetic terms linear in time derivatives - do not substantially affect our arguments. It also happens that the results for the non-relativistic systems of practical interest can be read off directly from

\footnotetext{
$\S$ Appendix A briefly reviews our conventions concerning this statistics field.
} 
the relativistic answers using the trick outlined in Appendix B.

- Quasi-particle Lagrangian: $\mathcal{L}_{p}(\xi, a+A)$ is the (firstquantized) lagrangian which describes the quasi-particle motion and their coupling to the electromagnetic field, $A_{\mu}$. A first-quantized representation is chosen because this makes the duality between particles and vortices most transparent later on. The detailed form of this lagrangian is not important in what follows, apart from the form of the coupling to the gauge field, $(a+A)_{\mu}$, whose form is important, but which follows on grounds of gauge invariance. To be concrete, in the absence of other interactions the particle lagrangian might be given explicitly by

$$
\mathcal{L}_{p}=\sum_{k}\left[\frac{m}{2} \dot{\xi}_{k}^{\mu} \dot{\xi}_{k \mu}-q_{k} \dot{\xi}_{k}^{\mu}(a+A)_{\mu}\right] \delta\left[x-\xi_{k}(t)\right],
$$

where $q_{k}$ here denotes the quasi-particle charge, normalized so that $q_{k}=-1$ for electrons.

- Dependence on External Parameters: All of the dependence on the external variables, like magnetic field, $B$, enter through the parameters of the effective lagrangian. For instance, in the example where the quasi-particle dynamics is described by eq. (3) they would enter through the quasi-particle mass, $m$, and the parameter $\kappa$, although the dependence would be more involved for more complicated quasi-particle dynamics. The same is almost true for the dependence on temperature, $T$. That is, the temperature dependence contributed when integrating out high-energy modes is embedded in the system parameters, but there is also additional temperature dependence associated with the integration over the low-energy degrees of freedom themselves, such as $\xi_{k}^{\mu}$. Both forms of temperature dependence are included in our later discussions of the temperature-dependence of particle-vortex duality.

- The Goldstone Variable: If the difference between the number of vortices and anti-vortices, $N_{v}$, is nonzero then there is a complex order parameter somewhere in the system which typically vanishes at the positions of the vortices (and anti-vortices), and takes a nonzero value asymptotically far away. The number $N_{v}$ is then related to the winding of the phase of this order parameter around a circle which encloses all of the vortex positions. The field $\phi$ in eq. (2) represents the phase of this order parameter. We assume, in writing eq. (2), that the order parameter carries nonzero electric charge, $q_{\phi} \neq 0$, and so it spontaneously breaks the electromagnetic $U(1)$ gauge group. The scale of the parameter $\kappa$ is of order of the scale of the symmetry-breaking expectation value.

Since the quanta of $\phi$ are the Goldstone bosons for the assumed symmetry breaking, $\phi$ is guaranteed to be in the low-energy theory. Indeed $\phi$ would be responsible for the long-range interactions experienced between vortices, if $q_{\phi}$ were zero. Furthermore, its couplings to $(a+A)_{\mu}$ are dictated by gauge invariance. Finally, because it is a Goldstone variable $\phi$ is guaranteed by Goldstone's theorem to decouple at low energies (modulo the usual Coleman-Mermin-Wagner caveats), thereby justifying its semi-classical treatment using a derivative expansion [26]. The ellipses in eq. (2) represent all of the other effective interactions obtained when all higher degrees of freedom are integrated out. Since these all involve inverse powers of the higher-energy scales, such as the order parameter scale, they are irrelevant for the present purposes compared to those explicitly displayed.

In the derivation of KLZ [12] this order parameter was the bosonic field which described the electrons, but for the present purposes it could equally well be a bilinear of fermion fields, or something still more complicated. All we need assume is that the order parameter spontaneously breaks electromagnetic gauge invariance, and that its boundary conditions at spatial infinity incorporate the winding corresponding to vortex number $N_{v}$.

\section{B. A Dual Description of the Vortices}

In order to better display the particle-vortex duality, it is convenient to use the dual description of the vortex degrees of freedom 27 29. We here implement this duality transformation by recognizing that it is a special case of a general dualization algorithm 30 (which also has applications to bosonization in one and two dimensions [31]), and is derived in detail for the present system in Appendix C. (We repeat the derivation in a secondquantized format in Appendix D, using the same model - the nonrelativistic abelian Higgs model supplemented with Chern Simons coupings - used by KLZ.)

The result, when applied to the quasi-particle/vortex action, eq. (2), is:

$$
\begin{aligned}
\tilde{\mathcal{L}}_{\text {eff }}= & -\frac{\pi}{2 \theta} \epsilon^{\mu \lambda \nu} a_{\mu} \partial_{\lambda} a_{\nu}-\epsilon^{\mu \lambda \nu} b_{\mu} \partial_{\lambda}\left(a_{\nu}+A_{\nu}\right) \\
& +\mathcal{L}_{p}(\xi, a+A)+\mathcal{L}_{v}(y, b)+\ldots,
\end{aligned}
$$

where:

- The field $b_{\mu}$ : is the new (gauge potential) field which is the dual representation of the Goldstone field, $\phi$. It carries all of the information about the long-range interactions amongst the vortices.

- The variables $y_{a}^{\mu}(t)$ : label the positions of the centres of the vortices and anti-vortices defined, for instance, as the positions of the zeros of the underlying order parameter whose phase was $\phi$. As is seen in Appendix C, these positions naturally arise as variables during the duality transformation once one takes into account the boundary conditions satisfied by $\phi$ in the presence of vortices.

- The Vortex Lagrangian $\mathcal{L}_{v}(y, b)$ : describes the dynamics of the vortices and their couplings to the field $b_{\mu}$. 
Although this action can be complicated, reflecting the potentially complicated dynamics of vortices in the material of interest, it must have at least the following two terms:

$$
\mathcal{L}_{v}=\sum_{a}\left[\frac{M}{2} \dot{y}_{a}^{\mu} \dot{y}_{a \mu}-\frac{2 \pi N_{a}}{q_{\phi}} \dot{y}_{a}^{\mu} b_{\mu}\right] \delta\left[x-y_{a}(t)\right],
$$

where $N_{a}$ denotes the vorticity (or winding number) of each vortex. The coupling term between $y_{a}^{\mu}$ and $b_{\mu}$ falls directly out of the duality transformation, and so is quite generally known. It should be noticed that although the kinetic term for $y_{a}^{\mu}$ does not itself follow directly by dualizing eq. (2), its form is fixed quite generally from symmetry arguments [32]. This is because the $y_{a}^{\mu}$ 's may themselves be thought of as Goldstone bosons for the breaking of translation invariance, which is here broken by the positions of the vortices themselves. In general there may also be other interactions to supplement eq. (5), which describe the interactions of the vortices with other degrees of freedom, such as disorder.

- A $b_{\mu}$ kinetic term: of the form $-\frac{1}{4 \kappa q_{\phi}^{2}} f_{\mu \nu}^{b}\left(f^{b}\right)^{\mu \nu}$, with $f_{\mu \nu}^{b}=\partial_{\mu} b_{\nu}-\partial_{\nu} b_{\mu}$, is also produced when performing the dualization, but is not written in eq. (4). It has been dropped since it is inversely proportional to $\kappa$, which is one of the high-energy scales whose inverse we are ignoring in the low-energy, long-wavelength limit. It would compete with a term proportional to $\left(f_{\mu \nu}+F_{\mu \nu}\right)\left(f^{\mu \nu}+F^{\mu \nu}\right)$ (where $f_{\mu \nu}$ and $F_{\mu \nu}$ are the field strengths for $a_{\mu}$ and $A_{\mu}$ ), which was among the ellipses appearing in eq. (2). (Alternatively, both such terms may be absorbed into the general response function which is obtained when $\xi_{k}^{\mu}$ and $y_{a}^{\mu}$ are integrated out, as we are shortly to describe.)

- Equivalence: It is to be emphasized that eq. (代) is just a change of variables of eq. (2), and so describes precisely the same physics. In particular, both expressions reproduce precisely the same electromagnetic response once all degrees of freedom except for $A_{\mu}$ are integrated out:**

$$
\begin{gathered}
e^{i \Gamma(A)=}=\int\left[d a_{\mu}(x)\right] \prod_{k}\left[d \xi_{k}^{\mu}(t)\right] \exp \left[i \int d^{3} x \mathcal{L}_{\mathrm{eff}}(\xi, a, A)\right] \\
=\int\left[d a_{\mu}(x)\right]\left[d b_{\mu}(x)\right] \prod_{a}\left[d y_{a}^{\mu}(t)\right] \\
\times \exp \left[i \int d^{3} x \tilde{\mathcal{L}}_{\mathrm{eff}}(y, a, b, A)\right] .
\end{gathered}
$$

\footnotetext{
${ }^{* *}$ Although we present our results for real time and at zero temperature, our duality relations can be derived equally well in imaginary time at nonzero temperature.
}

\section{PARTICLE-VORTEX DUALITY}

The beauty of eqs. (3), (4) and (5) is that they display the quasi-particle and vortex degrees of freedom in a way which emphasizes the similarity of the particles and vortices. For instance, if other interactions are negligible, so the particle and vortex dynamics is given by eqs. (3) and (5), and in the absence of the Chern-Simons term involving $\theta$, eq. (4) has the symmetry with respect to the interchanges $\xi_{k}^{\mu} \leftrightarrow y_{a}^{\mu}, b_{\mu} \leftrightarrow a_{\mu}+A_{\mu}$, so long as the quasi-particle masses and charges are also interchanged: $q_{k} \leftrightarrow 2 \pi N_{a} / q_{\phi}$ and $m \leftrightarrow M$. The same is also true if $\mathcal{L}_{p}$ and $\mathcal{L}_{v}$ are more complicated, provided that the additional complications treat particles and vortices democratically, by contributing terms of the same form to both $\mathcal{L}_{p}$ and $\mathcal{L}_{v}$.

We may now be more precise as to what is meant by dual systems. Given a two-dimensional system whose EM response is governed by $n$ quasi-particles (having mass $m$ etc) and $N$ vortices (having mass $M$ etc), we define the dual to be the system having $N$ quasi-particles (with mass $M$ etc) and $n$ vortices (of mass $m$ etc). The cases of real interest for the applications which follow are the cases involving particles only or vortices only: $n=0$ and $N=0$.

\section{A. Expressions for the EM Response}

We now wish to determine how the electromagnetic response of dual systems are related to one another. To do so, imagine integrating out the quasi-particles and vortices by performing the path integral over their positions, $\xi_{k}^{\mu}$ and $y_{a}^{\mu}$. If we are only interested in linear response, we need not actually evaluate these integrals, but may parameterize them in terms of response functions, $P^{\mu \nu}$, and $V^{\mu \nu}$, as follows. That is, if we define:

$$
\begin{aligned}
e^{i \gamma_{p}(a+A)} & =\int \prod_{k}\left[d \xi_{k}^{\mu}(t)\right] \exp \left[i \int d^{3} x \mathcal{L}_{p}(\xi, a+A)\right] \\
e^{i \gamma_{v}(b)} & =\int \prod_{a}\left[d y_{a}^{\mu}(t)\right] \exp \left[i \int d^{3} x \mathcal{L}_{v}(y, b)\right],
\end{aligned}
$$

then for linear response it suffices to take:

$$
\begin{aligned}
& \gamma_{p}(a)=-\frac{1}{2} \int d^{3} x d^{3} x^{\prime} a_{\mu}(x) P^{\mu \nu}\left(x-x^{\prime}\right) a_{\nu}\left(x^{\prime}\right), \\
& \gamma_{v}(b)=-\frac{1}{2} \int d^{3} x d^{3} x^{\prime} b_{\mu}(x) V^{\mu \nu}\left(x-x^{\prime}\right) b_{\nu}\left(x^{\prime}\right),
\end{aligned}
$$

where $P^{\mu \nu}\left(x-x^{\prime}\right)$ and $V^{\mu \nu}\left(x-x^{\prime}\right)$ define the particle and vortex response functions. Notice that for dual systems we expect $P^{\mu \nu}=\tilde{V}^{\mu \nu}$ and $V^{\mu \nu}=\tilde{P}^{\mu \nu}$, where the tilde denotes the result evaluated in the dual system. 
If eqs. (17) - (10) are used in (ब), then the remaining integrals over $a_{\mu}$ and $b_{\mu}$ are Gaussian and so may be evaluated explicitly to obtain the electromagnetic response function, defined by:

$$
\Gamma(A)=-\frac{1}{2} \int d^{3} x d^{3} x^{\prime} A_{\mu}(x) \Pi^{\mu \nu}\left(x-x^{\prime}\right) A_{\nu}\left(x^{\prime}\right) .
$$

One might be queasy about the consistency of first expanding to quadratic order in $b_{\mu}$ and then integrating $b_{\mu}$ over all values, and this queasiness would be justified if the expansion to quadratic order was done because $b_{\mu}$ is small. Such a calculation really presumes an effectivefield theory approach, where all terms involving higher powers of fields are irrelevant (in the RG sense), and so may be neglected within the very low energy effective theory. This is true in the present instance so long as anomalous dimensions are small, so that relevance may be judged purely using naïve dimensional analysis. It is here that we implicitly assume the quasi-particles and vortices to be weakly coupled to other degrees of freedom, although this does not also imply weak coupling for the underlying electrons. In any case, we later provide an alternative derivation of a subset of our results which does not rely on the quadratic approximation of eqs. (9), (10).

We believe that our treatment of the quasi-particles purely within an effective-theory framework represents an important conceptual difference between the arguments formulated here, and those presented in the spirit of KLZ, in Appendix D. They differ because in the KLZ framework the bosonic field whose phase describes the vortices is taken to be a direct description of the underlying electrons, rather than a low-energy effective field. As a result a weak-coupling mean-field analysis for the KLZ field is more directly tied to the strength of the couplings of the underlying electrons.

Before quoting the result obtained in this way for the EM response, it is worth first defining some notation. The polarization tensor is usually taken to have the standard rotationally-invariant and gauge-invariant but nonrelativistic form:

$$
\begin{aligned}
\Gamma(A)=\frac{1}{2} \int & d^{3} x d^{3} x^{\prime}\left[\pi_{1}\left(x-x^{\prime}\right) E_{i}(x) E_{i}\left(x^{\prime}\right)\right. \\
& -\pi_{2}\left(x-x^{\prime}\right) B(x) B\left(x^{\prime}\right) \\
& -\pi_{3}\left(x-x^{\prime}\right) \epsilon^{\mu \nu \lambda} A_{\mu}(x) \partial_{\nu} A_{\lambda}\left(x^{\prime}\right),
\end{aligned}
$$

defining the electromagnetic form factors, $\pi_{1}, \pi_{2}$ and $\pi_{3}$. In what follows our main interest is in $\pi_{1}$ and $\pi_{3}$, which control the conductivities $\sigma_{x x}$ and $\sigma_{x y}$. Because of this, and because of the greater simplicity of the resulting formulae, we specialize instead to the relativistic version of eq. (12), which we write in momentum space as:

$$
\Pi^{\mu \nu}=\Pi_{1}\left(p^{2}\right) \Lambda^{\mu \nu}+\Pi_{3}\left(p^{2}\right) J^{\mu \nu},
$$

where $\Lambda^{\mu}{ }_{\nu}$ and $J^{\mu \nu}$ are defined by $\Lambda^{\mu}{ }_{\nu}=\delta_{\nu}^{\mu}-p^{\mu} p_{\nu} / p^{2}$ and $J^{\mu \nu}=i \epsilon^{\mu \lambda \nu} p_{\lambda} / \sqrt{p^{2}}$. Because the form factors of eqs. (12) and (13) are related (in momentum space) by $\Pi_{1}=(p / \hbar)^{2} \pi_{1}=(p / \hbar)^{2} \pi_{2}$ and $\Pi_{3}=\sqrt{p^{2} / \hbar^{2}} \pi_{3}$, the relativistic form is sufficient to follow how the quantities $\pi_{1}$ and $\pi_{3}$ transform under duality transformations. (More generally, results for the generic case $\pi_{1} \neq \pi_{2}$, are easily obtained using the trick described in Appendix B.)

The great utility of the relativistic expression, eq. (13), follows because the tensors $\Lambda^{\mu}{ }_{\nu}$ and $J^{\mu \nu}$ satisfy the identities: $\Lambda_{\alpha}^{\mu}{ }_{\alpha}^{\alpha}{ }_{\nu}=\Lambda_{\nu}^{\mu}, \Lambda_{\alpha}^{\mu} J^{\alpha \nu}=J^{\mu \alpha} \Lambda_{\alpha}^{\nu}=J^{\mu \nu}$ and $J^{\mu \alpha} J_{\alpha \nu}=-\Lambda_{\nu}^{\mu}$. Since the tensors $\Lambda$ and $J$ are related to one another in the same way as are the bases, 1 and $i$, of complex numbers, tensor manipulations with $\Pi^{\mu \nu}$ can be greatly simplified by re-expressing it as a complex variable:

$$
\Pi=\Pi_{1}+i \Pi_{3} .
$$

With this notation, and defining similar expressions for the complex quantities $\mathbf{P}$ and $\mathbf{V}$ in terms of the corresponding form factors $P_{1}, P_{3}, V_{1}$ and $V_{3}$, we find the result of integrating the fields $a_{\mu}$ and $b_{\mu}$ out of eq. (4) to be

$$
\boldsymbol{\Pi}=i \sqrt{\bar{p}^{2}}\left(\frac{\pi}{\theta}\right) \frac{\bar{p}^{2}+\mathbf{V} \mathbf{P}}{\bar{p}^{2}+\mathbf{V}\left(\mathbf{P}+i \sqrt{\bar{p}^{2}}\left(\frac{\pi}{\theta}\right)\right)}
$$

as the general relation between the EM response and the particle and vortex response functions. To avoid explicit factors of $\hbar$ we have defined $\bar{p}=p / \hbar$ in this expression. (The details of these integrations are given in Appendix E.) This expression is the main result on which our later conclusions are based.

We now record the special cases of this expression which will be used in the following sections.

- Addition of $2 \pi$ Flux: Since $\theta$ represents the statistics of the quasi-particles, the choice $\theta=2 k \pi$, with $k$ an integer, can have no physical effects: $\Pi(\theta+2 k \pi) \approx \Pi(\theta)$. Inspection of eq. (15) shows the implications of this statement for the EM response:

$$
\frac{1}{\Pi(\theta)} \approx \frac{1}{\Pi(\theta+2 k \pi)}=\frac{1}{\Pi(\theta)}-\frac{2 k i}{\sqrt{\bar{p}^{2}}},
$$

which reproduces a well-known result 12, 33.

- Quasi-particles Only: Should there be no vortices participating in the EM response at all (which, because of the duality transformation from $\phi$ to $b_{\mu}$, is equivalent to $\mathbf{V} \rightarrow \infty$ ), then eq. (15) simplifies to:

$$
\Pi=i \sqrt{\bar{p}^{2}}\left(\frac{\pi}{\theta}\right) \frac{\mathbf{P}}{\mathbf{P}+i \sqrt{\bar{p}^{2}}\left(\frac{\pi}{\theta}\right)} .
$$

which reduces to $\Pi=\mathbf{P}$ when $\theta \rightarrow 0$, corresponding to bose statistics for the charge-carrying quasi-particles. 
The corresponding result for fermionic charge carriers is similarly found by choosing $\theta= \pm \pi$.

- Vortices Only: In the event that only vortices are involved in charge transport, expression (15) reduces to:

$$
\boldsymbol{\Pi}=\frac{\bar{p}^{2}}{\mathbf{V}-i \sqrt{\bar{p}^{2}}\left(\frac{\theta}{\pi}\right)} .
$$

This also reduces to the usual result, $\boldsymbol{\Pi}=\bar{p}^{2} / \mathbf{V}$, for bosonic charge carriers, for which $\theta \rightarrow 0$.

\section{SOME CONSEQUENCES OF PARTICLE-VORTEX DUALITY}

In this section we derive the implications of particlevortex duality for the electromagnetic response of twodimensional systems.

We are interested in the situation where quasi-particles only or vortices only are responsible for charge transport, in which case a very simple expression may be derived for the action of particle-vortex duality on the EM response function, $\boldsymbol{\Pi}$. This may be derived by using eq. (17) to relate $\boldsymbol{\Pi}$ to $\mathbf{P}$ for the original system, and using eq. (18) to relate $\tilde{\boldsymbol{\Pi}}$ to $\tilde{\mathbf{V}}=\mathbf{P}$ for the dual system. Eliminating $\mathbf{P}$ from these two expressions gives the desired relation

$$
\frac{\tilde{\Pi}}{\bar{p}^{2}}=\frac{i \sqrt{\bar{p}^{2}}\left(\frac{\pi}{\theta}\right)-\Pi}{\bar{p}^{2}+i \sqrt{\bar{p}^{2}}\left(\frac{\pi}{\theta}+\frac{\theta}{\pi}\right) \Pi}
$$

directly expressing the dual response function, $\tilde{\boldsymbol{\Pi}}$, in terms of that of the original system.

The physical interpretation of expression (19) is obtained by using the connection between the small- $p^{2}$ limit of the form factors, $\Pi_{1}$ and $\Pi_{3}$, and measurable electromagnetic quantities. There are two main cases to consider:

\section{A. Superconductors and Insulators}

If there there is a gap at the relevant part of the spectrum, then one expects the EM response function to be analytic in momentum space. This implies the functions $\Pi_{1}$ and $\Pi_{3}$ have the following small- $p^{2}$ form;

$$
\Pi_{1}=A_{1}+B_{1} \bar{p}^{2}+\ldots, \quad \Pi_{3}=\sqrt{\bar{p}^{2}}\left(A_{3}+\ldots\right) .
$$

The $\sqrt{\bar{p}^{2}}$ does not conflict with $\Pi^{\mu \nu}$ being analytic as $p^{\mu} \rightarrow 0$ because it cancels a similar factor in the definition of the tensor $J^{\mu \nu}$. The constant $A_{1}$ is nonzero only for superconductors, with $A_{1}$ inversely related to the medium's electromagnetic penetration depth. If $A_{1}=0$ then the material is an insulator and $B_{1}$ is related to the dielectric response of the medium. The constant $A_{3}$ corresponds to a Hall conductivity for the system, which may vanish but need not.

There are two important sub-cases to consider:

\section{Superconductors}

If $A_{1}$ is nonzero, then the material is a superconductor and $A_{1}=m_{\gamma}^{2}=1 / \lambda$ is the corresponding photon squared mass, or inverse penetration length. In this case there is a gap because of the Anderson-Higgs mechanism.

Inserting assumption (20) for $\Pi$ (with $A_{1} \neq 0$ ) into the duality expression, eq. (19), implies $\tilde{\Pi}$ also has an expansion of the form (20), with:

$$
\begin{aligned}
\tilde{A}_{1} & =0 \\
\tilde{A}_{3} & =\frac{1}{\frac{\theta}{\pi}+\frac{\pi}{\theta}} \\
\tilde{B}_{1} & =\frac{1}{\left[1+\left(\frac{\theta}{\pi}\right)^{2}\right]^{2} A_{1}} .
\end{aligned}
$$

We see that the system dual to a superconductor is an insulator, whose Hall conductivity and dielectric function are related to the superconducting penetration length and the statistics of its quasi-particle charge carriers.

- Bosonic Charge Carriers: A particularly interesting special instance of expression (21) is the case of bosonic charge carriers (such as Cooper pairs), for which $\theta=0$. In this case we find as dual a dielectric with vanishing Hall conductivity and dielectric function given by $\tilde{B}_{1}=1 / A_{1}$. This is as expected on physical grounds I1] since the condensation of vortices in the dual system should produce an insulator. Notice that eq. (21) makes specific predictions as to the dependence of the dual dielectric function, $\tilde{B}_{1}$, as a function of temperature since it is related to the temperature dependence of the penetration length in the superconducting system.

- Fermionic Charge Carriers: If we instead choose $\theta=$ $\pi$, as is appropriate for fermion charge carriers, we find the dual system has no Ohmic resistance, but has Hall conductivity $\sigma_{x y}=\frac{1}{2}$.

\section{Insulators}

If $A_{1}=0$ then the material is an insulator and the gap exists because of the absence of low-energy charged quasi-particles which carry current. In this case $B_{1}$ is related to the dielectric response of the medium and the duality transformation produces the following small- $p^{2}$ EM response:

$$
\begin{aligned}
& \tilde{A}_{1}=0, \\
& \tilde{A}_{3}=\frac{\frac{\pi}{\theta}-A_{3}}{1-\left(\frac{\theta}{\pi}+\frac{\pi}{\theta}\right) A_{3}}, \\
& \tilde{B}_{1}=\frac{B_{1}\left(\frac{\pi}{\theta}\right)^{2}}{\left[1-\left(\frac{\theta}{\pi}+\frac{\pi}{\theta}\right) A_{3}\right]^{2}} .
\end{aligned}
$$


We see that the image of an insulator is another insulator, although with different Hall conductivity and dielectric function.

\section{B. Conductors}

For conductors the form factor $\Pi_{1}$ is not analytic as $p^{2} \rightarrow 0$. The limiting form for small $p^{2}$ is related to the conductivities by:

$$
\begin{aligned}
& \Pi_{1}\left(\bar{p}^{2}\right) \rightarrow \sigma_{x x} \sqrt{\bar{p}^{2}}+\ldots, \\
& \Pi_{3}\left(\bar{p}^{2}\right) \rightarrow \sigma_{x y} \sqrt{\bar{p}^{2}}+\ldots,
\end{aligned}
$$

and so the complex quantity $\Pi$ is related to the complex conductivity, $\sigma=\sigma_{x y}+i \sigma_{x x}$, by $\Pi=i \sqrt{\bar{p}^{2}} \sigma^{*}$.

In this case the duality transformation, eq. (19), preserves the momentum dependence of the form factors, implying $\tilde{\boldsymbol{\Pi}}=i \sqrt{\bar{p}^{2}} \tilde{\sigma}^{*}$, with the dual conductivities given by the holomorphic relation:

$$
\tilde{\sigma}=\frac{\frac{\pi}{\theta}-\sigma}{1-\left(\frac{\theta}{\pi}+\frac{\pi}{\theta}\right) \sigma} .
$$

- Fermion Charge Carriers: If we assume the original charge carriers to be fermions, as would be appropriate for the integer Quantum Hall systems, then eq. (24) reduces to the expression:

$$
\tilde{\sigma}=\frac{1-\sigma}{1-2 \sigma},
$$

which is the transformation which has been recognized [15] to imply the experimentally observed duality transformations $\rho_{x x} \rightarrow 1 / \rho_{x x}$ in the transitions between the $\sigma_{x y}=1$ Hall plateaux and the Hall insulator.

Duality relations between more complicated Hall plateaux may be similarly obtained by starting from the appropriate anyonic charge carriers, but the same result may be obtained more simply by combining eq. (25) with transformation (16) (which expresses the the absence of content of a shift of the statistics parameter by $2 \pi$ ) specialized to conductors:

$$
\sigma \approx \frac{\sigma}{1-2 \sigma}
$$

Transformations (25) and (26) together generate the group, $\Gamma_{0}(2)$, which is known to relate all of the allowed (odd-denominator) Hall states to one another, and so produces the anyonic Quantum Hall duality relations from the simpler fermionic one we have just considered.

- Boson Charge Carriers: Specializing eq. (24) to the case $\theta=0$ also gives a simple relation between the dual conductivities:

$$
\tilde{\sigma}=-\frac{1}{\sigma} .
$$

This transformation, together with eq. (26), also generates an infinite group, denoted $\Gamma_{\theta}(2)$, which relates dual conductors with bosonic charge carriers (such as superconducting films or Josephson junction arrays).

\section{Duality and Flow}

To this point we have found how the EM response of dual systems are related to one another. We wish now to understand how the duality transformations change as external parameters like magnetic field and temperature are continuously varied. The question of how systems change as external variables are varied is particularly sharp for conductors, since in this case both the original system and its dual are of the same type (i.e. they are both conductors). If one imagines a system tracing out a curve in the conductivity plane as $T$ and $B$ are varied, the position of the dual system traces out another curve in the same plane. We wish to argue that the resulting flow commutes with the action of the two duality transformations, eqs. 25) and (26) (or (26) and (27)), in the conductivity plane.

The magnetic field strength, and other other microscopic properties, enter into the above arguments only by changing the values which are taken by the masses and other parameters appearing in the effective lagrangian, eqs. (2) and (4). Temperature partly appears in the same way, but also appears in the integration over the low-energy degrees of freedom (such as by rotating to euclidean signature and imposing periodicity in imaginary time). Now comes the main point. All of the consequences of duality follow from the statements $P^{\mu \nu}=\tilde{V}^{\mu \nu}$ and $V^{\mu \nu}=\tilde{P}^{\mu \nu}$, which relate the response functions of dual systems (or the analogous statement expressing the addition of $2 \pi$ statistics flux). And these statements are true for all values of magnetic field, temperature etc., so long as these quantities do not introduces differences in form between the particle and vortex lagrangians, $\mathcal{L}_{p}$ and $\mathcal{L}_{v}$.

Suppose we now consider a system of quasiparticles, $S\left(T_{0}, B_{0}\right)$, chosen for some specific temperature, $T_{0}$, and magnetic field, $B_{0}$, involving $N$ particles of mass $m$ and charge $q$. Next, suppose that $\tilde{B}_{0}$ and $\tilde{T}_{0}$ are chosen to produce the dual system, $\tilde{S}$, having $N$ vortices, also with mass $m$ and vortex charge $q$. That is: $S\left(\tilde{T}_{0}, \tilde{B}_{0}\right)=\tilde{S}\left(T_{0}, B_{0}\right)$. Notice that the existence of such a $\tilde{T}_{0}$ and $\tilde{B}_{0}$ is plausible given that it involves the solution of two equations for two unknowns. For instance, for particle-vortex interchange the two equations are: $M\left(\tilde{T}_{0}, \tilde{B}_{0}\right)=m\left(T_{0}, B_{0}\right)$ and $Q\left(\tilde{T}_{0}, \tilde{B}_{0}\right)=q\left(T_{0}, B_{0}\right)$, where $M$ and $Q$ are the functions of $T$ and $B$ which define the vortex mass and charge.

Now imagine changing the magnetic field and/or temperature, say to $B^{\prime}=B_{0}\left(1+\delta_{B}\right)$ and $T^{\prime}=T_{0}\left(1+\delta_{T}\right)$. 
The question we ask is this: if we change the dual system by the same amount, to $\tilde{B}^{\prime}=\tilde{B}_{0}\left(1+\delta_{B}\right)$ and $\tilde{T}^{\prime}=\tilde{T}_{0}\left(1+\delta_{T}\right)$, is the resulting system still dual to the first? That is, is $S\left(\tilde{T}^{\prime}, \tilde{B}^{\prime}\right)=\tilde{S}\left(T^{\prime}, B^{\prime}\right)$ ? The answer is 'yes', because the question asked of both systems is the same: what is the change in response of a system of $N$ objects of mass $m$ and charge $q$ as $T$ and $B$ are varied? The only difference between the system and its dual is that for $S$ the $N$ objects are particles and for $\tilde{S}$ they are vortices.

Arguing in the same way for the attachment of $2 \pi$ statistics flux, we see that the entire duality group must commute with the flow through the conductivity plane as $B$ and $T$ are varied. For fermions this implies a $\Gamma_{0}(2)$ invariant flow, while for bosons $\Gamma_{\theta}(2)$-invariant flow is implied.

\section{Beyond Linear Response}

In this section we present a slightly different version of part of the previous section's derivation, whose aim is to express the action of particle-vortex duality on the electromagnetic response without relying on the linearresponse approximation, eqs. (9), (10). The argument we present assumes fermion charge carriers, since it relies on using a statistics parameter $\theta= \pm \pi$. Besides clarifying our derivation, we present this separate line of argument because we believe it will ultimately prove fruitful in its own right by explaining the experimental evidence [36] for current-voltage duality seen in Quantum Hall systems beyond the linear Ohm's Law approximation.

We pick up the story of section (III) just before making the linear-response approximations, eqs. (9), (10). The response function, $\Gamma(A)$, for the system of charged particles is given by functionally integrating the fields $a_{\mu}$ and $\xi_{k}^{\mu}$ weighted by the lagrangian density:

$$
\mathcal{L}(\xi, a, A)=+\frac{1}{2} \epsilon^{\mu \nu \lambda} a_{\mu} \partial_{\nu} a_{\lambda}+\mathcal{L}_{\text {kin }}(\xi)+j^{\mu}(\xi)(a+A)_{\mu},
$$

where we have chosen $\theta=-\pi$ and we need not be concerned about the detailed form of the particle kinetic term, $\mathcal{L}_{\text {kin }}(\xi)$, or current, $j^{\mu}(\xi)$.

On the other hand, the response function of the system related by particle-vortex duality to the original system involves integrating the dual lagrangian

$$
\begin{gathered}
\tilde{\mathcal{L}}(y, a, b, A)=-\frac{1}{2} \epsilon^{\mu \nu \lambda} a_{\mu} \partial_{\nu} a_{\lambda}-\epsilon^{\mu \nu \lambda} b_{\mu} \partial_{\nu}(a+A)_{\lambda} \\
+\tilde{\mathcal{L}}_{\text {kin }}(y)+\tilde{j}^{\mu}(y) b_{\mu},
\end{gathered}
$$

where we have instead chosen to represent fermionic statistics by choosing $\theta=+\pi$. In order to relate $\tilde{\Gamma}(A)$ to $\Gamma(A)$ we explicitly perform the Gaussian integration over $a_{\mu}$ in eq. (29) and shift $b_{\mu} \rightarrow b_{\mu}+A_{\mu}$ in the result, to get

$$
\begin{array}{r}
\tilde{\mathcal{L}}(y, b, A)=+\frac{1}{2} \epsilon^{\mu \nu \lambda} b_{\mu} \partial_{\nu} b_{\lambda}-\frac{1}{2} \epsilon^{\mu \nu \lambda} A_{\mu} \partial_{\nu} A_{\lambda} \\
+\tilde{\mathcal{L}}_{\text {kin }}(y)+\tilde{j}^{\mu}(y)(b+A)_{\mu} .
\end{array}
$$

To the extent that $\tilde{\mathcal{L}}_{\text {kin }}(y)$ and $\mathcal{L}_{\text {kin }}(\xi)$ (and $\tilde{j}^{\mu}(y)$ and $j^{\mu}(\xi)$ ) have the same form, eq. (30) differs from eq. (28) only by the term $-\frac{1}{2} \epsilon^{\mu \nu \lambda} A_{\mu} \partial_{\nu} A_{\lambda}$, which does not depend at all on the integration variables. Performing the remaining integrations therefore relates $\tilde{\Gamma}(A)$ to $\Gamma(A)$ by:

$$
\tilde{\Gamma}(A)=\Gamma(A)-\frac{1}{2} \int d^{3} x \epsilon^{\mu \nu \lambda} A_{\mu} \partial_{\nu} A_{\lambda},
$$

which is the main result of this section.

Once specialized to the linear response regime, eq. (31) implies the relation:

$$
\tilde{\Pi}^{\mu \nu}(p)=\Pi^{\mu \nu}(p)+i \epsilon^{\mu \lambda \nu} p_{\lambda},
$$

or: $\tilde{\sigma}_{x y}=\sigma_{x y}+1$. This agrees exactly with what is predicted for fermions by a particle-vortex transformation followed by a $2 \pi$ statistics shift, equation (25) followed (26).

\section{APPLICATIONS TO CONDUCTORS}

In this section we state some of the observable predictions which follow from the action of particle-vortex duality on the physical systems. We specialize in this section to the predictions for two kinds of conducting systems, those with fermionic charge carriers (or their images under repeated duality transformations) - corresponding to Quantum Hall systems - and those with bosonic charge carriers (or their duality images) - such as for superconducting thin films and/or Josephson junction arrays.

\section{A. Fermions: The Quantum Hall Effect}

We start with Quantum Hall systems, for which the results we derive are not new, having been derived from the the assumption of duality-invariant flows in ref. [15. We include this case anyway for three reasons. First, this paper strengthens the theoretical foundation of the assumption of duality-invariant flow, particularly away from the systems' critical points. Second, the experimental success of these predictions establishes the existence of systems which are clean enough for our quasi-particle/vortex effective theory to apply. Third, we may directly adapt many of the Quantum Hall results to the bosonic case, for which most of our predictions are new.

\section{Some Group Theoretical Facts}

In previous sections we have found the action of particle-vortex duality and $2 \pi$ statistics addition to both 
act on the complex conductivity in a fractional linear way, with integer coefficients. That is, we have found the transformations to be a subgroup of the group $\operatorname{PSL}(2, Z)$, defined by:

$$
\sigma \rightarrow \frac{a \sigma+b}{c \sigma+d}
$$

with $a, b, c$ and $d$ integers satisfying $a d-b c=1$. Any element of this group can be obtained as products of powers of the following two generators:

$$
S(\sigma):=-\frac{1}{\sigma}, \quad T(\sigma):=\sigma+1 .
$$

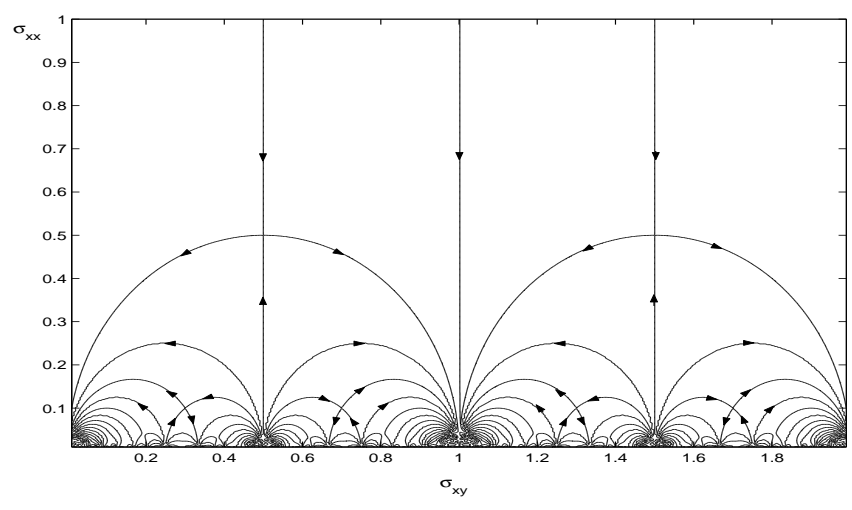

Figure 1: Flow lines for $\Gamma_{0}(2)$-invariant flow.

We have found the subgroup of this group which is relevant to Quantum Hall systems to be generated by:

$$
\begin{aligned}
& \sigma \rightarrow \frac{\sigma}{1-2 \sigma}=S T^{2} S(\sigma), \\
& \sigma \rightarrow \frac{1-\sigma}{1-2 \sigma}=T S T^{2} S(\sigma),
\end{aligned}
$$

or, equivalently, by $S T^{2} S$ and $T$. Any point in the upper complex $\sigma$-plane can be reached from a fundamental region, which we take to be the vertical strip between $\sigma=0$ and $\sigma=1$, with the interior of the disc with radius $=\frac{1}{2}$ centred at $\sigma=\frac{1}{2}$ removed. As we shall shortly see, the boundaries of this fundamental domain are quite generally flow lines for $\Gamma_{0}(2)$-invariant flow, and so may be seen in Fig. (1).

It turns out that the subgroup obtained from these two generators, denoted $\Gamma_{0}(2)$, is equivalent to that defined by requiring the coefficient $c$ in eq. (33) to be even [10,11, 13. This alternative definition shows that when acting on the real axis, $\sigma=\sigma^{*}$, it takes rational numbers to themselves, with odd-denominator fractions going to odd-denominator fractions, and even-denominator fractions mapping to even-denominator fractions. 门十

\footnotetext{
${ }^{\dagger \dagger}$ It is tantalizing that the same group, $\Gamma_{0}(2)$, plays a central role in the hierarchical structure of $N=2$ supersymmetric Yang-Mills theories 37.
}

\section{Predictions}

The following consequences follow from the condition that a flow in the $\sigma$-plane commutes with the group $\Gamma_{0}(2)$ :

1. Universal critical points 38 are predicted for the flow at any point, $\sigma_{\text {crit }}$, which is mapped to itself under any member of $\Gamma_{0}(2), \gamma\left(\sigma_{\text {crit }}\right)=\sigma_{\text {crit }}$, for which the denominator $c \sigma+d$ is neither zero or infinite 11, 13, 16, The complete set of such points occur at $\sigma_{\text {crit }}=\frac{1}{2}(1+i)$ and its images under $\Gamma_{0}(2)$. This prediction is borne out experimentally [39], since there is a one-to-one correspondence between the experimental critical points of the flow and the fixed points of the group. No experimental evidence exists for critical points not corresponding to fixed points of the group, although these could exist in principle.

2. Although the symmetry does not predict the critical exponents at the fixed points [19], these exponents must be the same for all fixed points which are related by $\Gamma_{0}(2)[11,13,16]$. This remarkable equivalence of critical exponents at different fixed points is known as super-universality, and was argued for on more model-dependent grounds in ref. 40. Historically, the experimental success of this 'prediction' 41,42 stimulated the search for an underlying symmetry group.

3. Exact flow lines in the $\sigma$ plane can be derived from $\Gamma_{0}(2)$ invariance plus invariance under particle-hole symmetry: $\sigma \rightarrow 1-\sigma^{*}$. The existence and shape of these flow lines depends only on these symmetries and not on any other details of the flow's $\beta$ function [15]. Figure (1) shows some of the flow lines predicted in this way, all of which are semi-circles or vertical lines in the $\sigma$-plane. The arrows indicate the direction of flow to the infrared (whose direction does not follow purely on symmetry grounds). This very general derivation of the 'semi-circle law' - which had been earlier predicted on more modeldependent grounds 43 - is spectacularly exhibited by experimental systems 44 .

4. Flow in the infrared is towards the real axis, terminating on the real axis at attractive fixed points at odd-denominator fractions. Even-denominator fractions similarly form repulsive fixed points of the flow. One finds in this way a robust explanation of the existence of odd denominator Hall plateaux.

5. Since all allowed transitions between Hall plateaux correspond to semi-circles which may be obtained by the action of $\Gamma_{0}(2)$ from the basic semi-circle connecting $\sigma=0$ to $\sigma=1$, one finds a selection 
rule which expresses which plateaux may be obtained from which by varying external parameters like magnetic field and temperature [14]. The selection rule obtained in this way states that a fraction $p_{2} / q_{2}$ can be reached by a quantum Hall transition from a fraction $p_{1} / q_{1}$, with $q_{1}$ and $q_{2}$ both odd integers, only if $\left|p_{1} q_{2}-p_{2} q_{1}\right|=1$. This agrees precisely with all the observed Hall sequences.

6 . There is an element of $\Gamma_{0}(2)$ which maps each of the flow lines to itself, with its ends reversed. For instance, the element $\gamma(\sigma)=(\sigma-1) /(2 \sigma-1)$, which we here identify as the expression of particle-vortex duality for fermions, does so for the semi-circle connecting $\sigma=0$ to $\sigma=1$. When specialized to transitions from Laughlin plateaux to the Hall insulator this symmetry element is precisely the experimentally observed [36] duality $\rho_{x x} \rightarrow 1 / \rho_{x x}$ with $\rho_{x y}$ fixed [15].

We regard the natural interpretation of the great experimental success of these predictions to be that the electromagnetic response of these systems is dominated by quasi-particles and vortices. Furthermore the systems are sufficiently clean to justify the neglect of those particle and vortex interactions which destroy the underlying particle-vortex duality, as described here. Finally, the relevant quasi-particles are in the fermionic equivalence class, in the sense that they are either fermions, or are obtainable from fermions by the action of $\Gamma_{0}(2)$.

There is also some evidence for a few Hall systems for which the critical conductivity is not at the universal values [44], and for direct plateau-insulator transitions which do not correspond to semi-circles as predicted here [45], and an understanding of why particle-vortex duality fails here would be very instructive. Since these typically involve samples with the most disorder, one possibility is that Landau-level mixing is not negligible in these systems [46,47. We believe this to an instance where interactions with the disorder ruin particle-vortex duality, and so destroy the underlying symmetry of the flow.

Another potential difficulty often raised in connection with this picture is the observed failure of scaling at very low temperatures in some samples [48] as one passes through the critical regime. We put these experiments aside, since although these are potentially very telling experiments, since scaling is an inevitable consequence of a vanishing $\beta$ function, it is not yet clear what their proper interpretation is, and indeed there are other experiments which appear to support scaling, 44] 团

There is nevertheless content in the above symmetry arguments, since these imply an entire suite of predic-

\footnotetext{
$\ddagger$ See, however, ref. [17] for an alternative interpretation of the behaviour near the critical points.
}

tions which must all hold together if particle-vortex duality is valid. So we predict that the above consequences of $\Gamma_{0}(2)$-invariant flow should come as a package, with the failure of some implying the failure of the others.

\section{B. Bosons: Superconducting Films}

A fascinating consequence of the generality of the particle-vortex duality arguments we present here is that they predict different, but equally striking, phenomena for the electromagnetic response of other clean twodimensional systems. In this section we describe these predictions for systems whose charge-carrying quasiparticles have bose statistics (or the image of bose statistics under a group which we here specify). These predictions should have practical applications to superconducting thin films and Josephson Junction arrays, and some of them have been anticipated [1,2, 4 , for the metalinsulating and superconductor-insulator transitions of these systems.

\section{More Group Theoretical Facts}

For bosonic charge carriers (and those related to these by duality) the action of particle-vortex duality and $2 \pi$ statistics addition is generated by the following two $P S L(2, Z)$ elements:

$$
\begin{aligned}
& \sigma \rightarrow \frac{\sigma}{1-2 \sigma}=S T^{2} S(\sigma), \\
& \sigma \rightarrow-\frac{1}{\sigma}=S(\sigma),
\end{aligned}
$$

or, equivalently, $S$ and $T^{2}$.

This group is called $\Gamma_{\theta}(2)$, and is equivalent to the condition that $a, d$ are both odd and $b, c$ are both even, or vice versa, in the fractional linear transformation, $(a \sigma+$ $b) /(c \sigma+d)$. Since bosons may be obtained from fermions by shifting their statistics by $\Delta \theta=\pi$, this group may be obtained from the Quantum Hall group, $\Gamma_{0}(2)$, by conjugating by $S T S(\sigma)=\sigma /(1-\sigma)$. Concretely: $g \in$ $\Gamma_{\theta}(2)$ implies $g=S T S h(S T S)^{-1}$, for some $h \in \Gamma_{0}(2)$. (The proof of this statement is easiest to see if the identity $(S T)^{3}=1$ is used.) The simplest way to extract the predictions of the group $\Gamma_{\theta}(2)$ is therefore to derive them from those of $\Gamma_{0}(2)$ by conjugating with $S T S$. (Or, since $S$ is in $\Gamma_{\theta}(2)$ anyway, we can equally well get $\Gamma_{\theta}(2)$ by conjugating $\Gamma_{0}(2)$ with $T S(\sigma)=1-\frac{1}{\sigma}$ rather than $S T S$.) In particular, it is convenient to choose the fundamental region to be the vertical strip between $\sigma=0$ and $\sigma=-1$, with the interior of the disc with radius $=\frac{1}{2}$ centred at $\sigma=-\frac{1}{2}$ removed, the boundaries of which again appear as particular flow lines in figure (2). 


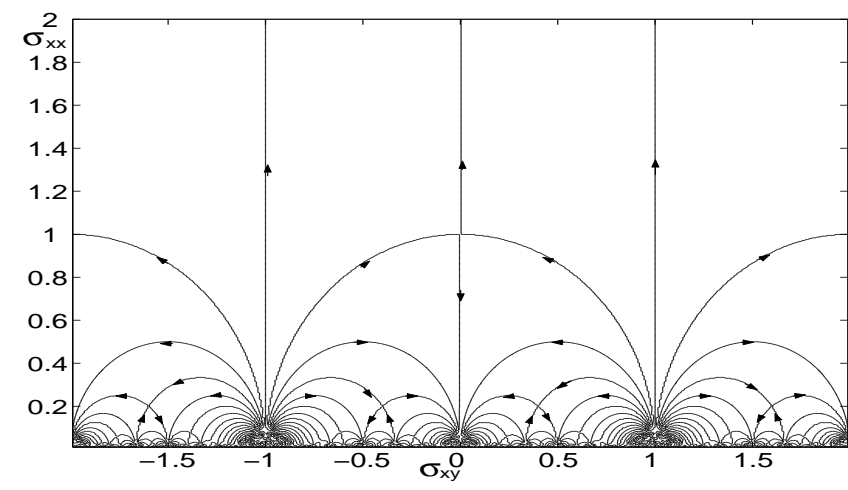

Figure 2: Flow lines for $\Gamma_{\theta}(2)$-invariant flow. Although this looks very much like Figure 1, careful examination shows different positions for the fixed points, and for the directions of flow along the lines.

\section{Predictions}

In this way we obtain the following consequences of the commuting of $\Gamma_{\theta}(2)$ with the flow in the $\sigma$-plane, which are the direct analogs of those described above for Quantum Hall systems.

1. Universal critical points are predicted for the flow at the fixed points of $\Gamma_{\theta}(2)$, which lie at $\sigma_{\text {crit }}=i$ and its images, $(b d+a c+i) /\left(c^{2}+d^{2}\right)$, under $\Gamma_{\theta}(2)$. The Ohmic conductivity is always 1 (odd integer) at these critical points while the Hall conductivity is even/odd. (These statements are for bosonic charge carriers with the same electric charge as an electron - these become $\sigma_{c r i t}=i q^{2}$ and its images if the bosonic charge carriers have charge $q$. In particular, the case $q= \pm 2$ applies if the bosons are Cooper pairs, such as considered in ref. [1].)

2. The critical exponents at all fixed points related by $\Gamma_{\theta}(2)$ must all be the same. In this way the results of the scaling theory of ref. [1] for the fixed point at $\sigma_{c r i t}=i$ may be extended to all of the other fixed points which are predicted to exist in the presence of magnetic fields.

3. Exact flow lines in the $\sigma$ plane are immediate consequences of $\Gamma_{\theta}(2)$ invariance and particle-hole symmetry, independent of the dynamical details of the flow's $\beta$ function. These are the images of the flow lines of Figure (1) under the conjugation by STS. The results are again semi-circles or vertical lines in the $\sigma$ plane, implying a new semi-circle law for these bosonic systems. (The semi-circle intersecting the critical point at $\sigma=i$ was anticipated in ref. [1].)
Figure (2) shows the flow lines which are predicted by the symmetry, with the flow directions given which follow from those of Figure (1) (which successfully describe Quantum Hall systems). Notice that the resulting flow on the imaginary axis agrees with the interpretation of $\sigma=i$ as a metal-insulator transition, with $\sigma_{x x}$ increasing or decreasing into the infrared on opposite sides of the transition.

4. More generally, for nonzero magnetic fields the flow in the infrared is towards the real axis, terminating on the attractive fixed points which are fractions, $\sigma=p / q$. The attractive fixed points of the flow therefore exhibit the fractional QHE, but with fractions for which $p q$ is even (as opposed to having $q$ odd, as was the case for fermions). Fractions with odd $p q$ are repulsive fixed points. In particular odd integers are repulsive while even integers are attractive.

5. There is a selection rule expressing which fractions may be obtained from which by varying external parameters like magnetic field and temperature. The selection rule obtained in this way states that fractions $p_{2} / q_{2}$ can be obtained from $p_{1} / q_{1}$ only if: (i) $p_{1}$ is odd and $q_{1}$ is even while $p_{2}$ is even and $q_{2}$ is odd (or vice versa with the subscripts 1 and 2 interchanged) and (ii) $\left|p_{1} q_{2}-p_{2} q_{1}\right|=1$.

6. There is an element of $\Gamma_{\theta}(2)$ which maps flow lines to themselves, with their ends reversed. For flow along the imaginary axis the element is simply $S(\sigma)=-1 / \sigma$, or $\sigma_{x x} \rightarrow 1 / \sigma_{x x}$, which is again the expression of particle-vortex duality for bosons.

We are led to predict the above startling properties for bosonic Quantum Hall systems, such as someday might be obtained from superconductor-insulator transitions in thin films, or from Josephson-junction arrays [50 54]. Unfortunately, these predictions cannot yet be tested with ordinary superconductors, because charge carrier densities and mobilities in these systems do not put them into the quantum Hall regime [55]. Their verification would be the smoking gun for particle-vortex duality, although its testing must wait until these systems can be reliably manufactured in the quantum regime. In this way particle-vortex duality might ultimately provide a connection between Quantum Hall systems and $\sigma_{x x} \rightarrow$ $1 / \sigma_{x x}$ as seen in metal-insulator transitions [56,57.

As in the case of Quantum Hall systems, these predictions require very few assumptions beyond the necessity to be in the quantum regime. First, the electromagnetic response must be dominated by quasi-particles and vortices, with the quasi-particles being bosons or related to these by $\Gamma_{\theta}(2)$ transformations. Second, the systems must be sufficiently clean to ensure the absence of interactions which distinguish the quasi-particles from vortices, 
and so ruin their similarity. Subject to these conditions all of the above consequences follow as a package from the quasi-particle-vortex duality symmetry of the flow in the conductivity plane.

\section{CONCLUSIONS AND OUTLOOK}

We have argued that a broad class of dual relationships arise in two dimensional systems for which the EM response is governed by particles and vortices whose properties are similar (perhaps because they are weakly interacting). For systems having fermions as the particles (or those related to fermions by the duality) the particlevortex duality implies the duality group is a level-two subgroup of $P S L(2, Z)$ called $\Gamma_{0}(2)$. We argue that this duality has been observed, since this group has been previously identified as explaining many of the observed properties of the EM response in Quantum Hall systems.

The generality of our arguments lead us to propose a similar, but distinct, set of properties for clean twodimensional systems whose EM response is controlled by bosons as the particles (or those related to bosons by the duality). The group implied in this case is another leveltwo subgroup of $P S L(2, Z)$ called $\Gamma_{\theta}(2)$. The observation of these specific predictions in these systems would be the definitive test of our ideas.

One might ask whether other duality symmetries apart from those described above might arise in other systems. More concretely, there are precisely five level-two subgroups of $S L(2, Z)$ [10], so one might wonder if other choices for quasi-particle statistics might generate these other three groups not yet used [58]. In fact, two of these groups can be ruled out as symmetries acting on the complex conductivity, because they do not contain the generator corresponding to the addition of $2 \pi$ statistics flux. However, because these groups are related to $\Gamma_{0}(2)$ and $\Gamma_{\theta}(2)$ by conjugating by $S$, they can be thought of as the action of these latter groups in the complex resistivity, rather than conductivity, plane. The third group, $\Gamma(2)$, is contained in the other four, and has been proposed elsewhere to play a role for Quantum Hall systems [59], in particular when the splitting between electron spins is much smaller than the gap between successive Landau levels 60.

\section{ACKNOWLEDGMENTS}

We thank M. Hilke, C.A. Lütken, M. Paranjape, F. Quevedo and S. Sondhi for helpful discussions. C.B. is grateful to the University of Barcelona, D.A.M.T.P at Cambridge University, and the Aspen Centre for Physics, and B.D. to the Groupe de Physique Théorique, IPN, Orsay, and Dept. de Fisica, CINVESTAV, Mexico City, Mexico for their kind hospitality while part of this work was carried out. Our research has been assisted by financial support from N.S.E.R.C. (Canada),
F.C.A.R. (Québec), the Ambrose Monell Foundation, CNRS (France) and Enterprise Ireland, Basic Research Grant no. SC/1998/739.

\section{APPENDIX A The Statistical Gauge Field}

In this appendix we review the concept of the statistical gauge field [25]. This serves not only to remind the reader of the construction, but also to set up the notation. This appendix is based on the review article by S-C. Zhang, 29.

\section{- First-Quantized Formulation:}

Consider a configuration of $N$ charged particles, each with the same charge $e$ and mass $m$ moving in a two dimensional plane, with positions $\mathbf{x}_{1}, \ldots, \mathbf{x}_{N}$ and separations $\mathbf{r}_{i j}=\mathbf{x}_{i}-\mathbf{x}_{j}=-\mathbf{r}_{j i}$. Allowing for particle-particle interactions, $e^{2} V\left(\mathbf{x}_{i}-\mathbf{x}_{j}\right)$, and the possibility of random static impurities giving, rise to an electric potential $U(\mathbf{r})$, the Hamiltonian for the system can be written as:

$$
\begin{gathered}
H=\frac{1}{2 m} \sum_{i}\left(-i \hbar \nabla_{\alpha}^{(i)}-e A_{\alpha}\left(\mathbf{x}_{i}\right)^{2}+e^{2} \sum_{i<j} V\left(\mathbf{x}_{i}-\mathbf{x}_{j}\right)\right. \\
+e \sum_{i} U\left(\mathbf{x}_{i}\right)
\end{gathered}
$$

where $\alpha=1,2$ and we use units in which the speed of light $c=1$. One can include a neutralising background field if desired, without changing any of the subsequent analysis significantly.

Following MacDonald [61, a gauge transformation to a new Hamiltonian is defined as follows. Let $\varphi_{i j}$ be the angle between the vector $\mathbf{r}_{i j}$ and an arbitrary fixed direction, e.g. the $x$-axis, so that $\varphi_{i j} \rightarrow \varphi_{i j}+\pi$ if the two particles $i$ and $j$ are interchanged. A gauge transformation from the multi-particle Schrödinger wave-function, $\psi\left(\mathbf{x}_{1}, \ldots, \mathbf{x}_{n}\right)$ to a new wave-function $\tilde{\psi}\left(\mathbf{x}_{1}, \ldots, \mathbf{x}_{n}\right)$ is defined as follows,

$$
\tilde{\psi}\left(\mathbf{x}_{1}, \ldots, \mathbf{x}_{N}\right)=\mathrm{e}^{i \frac{\theta}{\pi} \sum_{i<j} \varphi_{i j}} \psi\left(\mathbf{x}_{1}, \ldots, \mathbf{x}_{N}\right)
$$

for a constant $\theta$, as yet arbitrary. Under interchange of any two particles, $i$ and $j$, the phase factor changes by $\mathrm{e}^{i \theta}$. Thus, if $\theta=2 k \pi$ for some integer $k$, the phase factor is unity and the new wave function has exactly the same statistics as the old one - if it was symmetric under interchange of two particles before it remains so, if it was anti-symmetric under interchange of two particles before it remains so. If $\theta$ were an odd multiple of $\pi$ a bosonic wave function would be transformed into a fermionic one and vice versa.

The gauge transformation (38) can be incorporated into the vector potential $A_{\mu}, \mu=0,1,2$, for the electromagnetic field by defining a new field (the statistical gauge field) 


$$
a_{\alpha}\left(\mathbf{x}_{i}\right):=\frac{\hbar}{e} \frac{\theta}{\pi} \sum_{\{j ; j \neq i\}} \nabla_{\alpha}^{(i)} \varphi_{i j}
$$

where the gradient operator acts on the position of the $i$-th particle. The new gauge transformed Hamiltonian is then

$$
\begin{aligned}
\tilde{H}=\frac{1}{2 m} \sum_{i} & \left(-i \hbar \nabla_{\alpha}^{(i)}-e A_{\alpha}\left(\mathbf{x}_{i}\right)-e a_{\alpha}\left(\mathbf{x}_{i}\right)\right)^{2} \\
& +e^{2} \sum_{i<j} V\left(\mathbf{x}_{i}-\mathbf{x}_{j}\right)+e \sum_{i} U\left(\mathbf{x}_{i}\right) .
\end{aligned}
$$

It is stressed that the physics of this new Hamiltonian is identical to the old one, provided $\frac{\theta}{2 \pi}$ is an integer.

\section{- Second-Quantized Formulation:}

We now reformulate the above first quantised treatment in second quantised form, ignoring spin. Accordingly define,

$\tilde{\mathbf{H}}=$

$$
\begin{gathered}
\int d^{2} x \Psi^{\dagger}(\mathbf{x})\left[\frac{1}{2 m}(-i \hbar \underline{\nabla}-e \mathbf{A}(\mathbf{x})-e \mathbf{a}(\mathbf{x}))^{2}+e U(\mathbf{x})\right] \Psi(\mathbf{x}) \\
+\frac{1}{2} \int d^{2} x d^{2} x^{\prime} \delta \rho(\mathbf{x}) V\left(\mathbf{x}-\mathbf{x}^{\prime}\right) \delta \rho\left(\mathbf{x}^{\prime}\right)
\end{gathered}
$$

where $\rho(\mathbf{x})=e \Psi^{\dagger}(\mathbf{x}) \Psi(\mathbf{x})$ is the charge density and $\delta \rho=\rho-<\rho>$.

Equation (39) can be expressed as

$$
a_{\alpha}\left(\mathbf{x}_{i}\right)=-\left(\frac{\hbar \theta}{e \pi}\right) \sum_{j \neq i} \epsilon_{\alpha \beta} \frac{\left(\mathbf{r}_{i}-\mathbf{r}_{j}\right)^{\beta}}{\left|\mathbf{r}_{i}-\mathbf{r}_{j}\right|^{2}}
$$

which in continuum form is

$$
a_{\alpha}(\mathbf{x})=-\left(\frac{\hbar \theta}{e^{2} \pi}\right) \int d^{2} x^{\prime} \frac{\epsilon_{\alpha \beta}\left(x^{\beta}-x^{\prime \beta}\right)}{\left|\mathbf{x}-\mathbf{x}^{\prime}\right|^{2}} \rho\left(\mathbf{x}^{\prime}\right) .
$$

¿From this follows

$$
\epsilon^{\beta \alpha} \nabla_{\beta} a_{\alpha}(\mathbf{x})=\frac{2 \hbar \theta}{e^{2}} \rho(\mathbf{x})
$$

since $\nabla^{2} \ln \left(\left|\mathbf{x}-\mathbf{x}^{\prime}\right|\right)=2 \pi \delta^{(2)}\left(\left|\mathbf{x}-\mathbf{x}^{\prime}\right|\right)$. The curl of $\mathbf{a}$ is non-zero here, despite it's original definition as a gradient, because of Aharanov-Bohm type singularities in (39). These manifest themselves as $\delta$-function singularities in the curl of $\mathbf{a}$ in the first quantised theory but are spread into a smooth distribution on the right hand side of (44), in the second quantised theory. Equation (44) is a dynamical equation of motion for the statistical gauge field $a_{\mu}$, but it is not yet co-variant, as it lacks $a_{0}$ terms. To include these allow $a_{\alpha}$ and $\rho$ to depend on time, then differentiating (44) and expressing the result co-variantly gives

$$
\epsilon^{\beta \alpha} \partial_{\beta}\left(\partial_{0} a_{\alpha}-\partial_{\alpha} a_{0}\right)=\frac{2 \hbar \theta}{e^{2}} \dot{\rho}=-\frac{2 \hbar \theta}{e^{2}} \partial_{\alpha} j^{\alpha},
$$

where $j^{\alpha}$ is a current. This integrates to

$$
\epsilon^{\alpha \beta}\left(\partial_{0} a_{\beta}-\partial_{\beta} a_{0}\right)=-\frac{2 \hbar \theta}{e^{2}} j^{\alpha}
$$

This equation can be obtained, together with equation (44), by treating $a_{\mu}$ as a dynamical field in the action

$S=-\left(\frac{e^{2}}{4 \hbar \theta}\right) \int d t d^{2} x \epsilon^{\mu \nu \lambda} a_{\mu} \partial_{\nu} a_{\lambda}+\int d t d^{2} x a_{\mu} j^{\mu}$

which is the Chern-Simons action for the statistical gauge field coupled to a source.

The problem can now be re-written in path integral form. Define the original Lagrangian density for the matter fields

$$
\begin{aligned}
\mathcal{L}_{\Psi}= & \Psi^{\dagger}\left(i \hbar \partial_{t}-e A_{0}\right) \\
& -\Psi^{\dagger}\left[\frac{1}{2 m}(-i \hbar \underline{\nabla}-e \mathbf{A})^{2}-e U\right] \Psi \\
& -\frac{1}{2} \int d^{2} x^{\prime} \delta \rho(\mathbf{x}) V\left(\mathbf{x}-\mathbf{x}^{\prime}\right) \delta \rho\left(\mathbf{x}^{\prime}\right) .
\end{aligned}
$$

After introducing the statistical gauge field the new La(41grangian is

$$
\begin{aligned}
\tilde{\mathcal{L}}_{\Psi}= & \Psi^{\dagger}\left(i \hbar \partial_{t}-e\left(A_{0}+a_{0}\right)\right) \Psi \\
-\Psi^{\dagger} & {\left[\frac{1}{2 m}(-i \hbar \underline{\nabla}-e \mathbf{A}-e \mathbf{a})^{2}-e U\right] \Psi } \\
& -\frac{1}{2} \int d^{2} x^{\prime} \delta \rho(\mathbf{x}) V\left(\mathbf{x}-\mathbf{x}^{\prime}\right) \delta \rho\left(\mathbf{x}^{\prime}\right),
\end{aligned}
$$

together with the Chern-Simons Lagrangian

$$
\mathcal{L}_{a}=-\left(\frac{e^{2}}{4 \hbar \theta}\right) \epsilon^{\mu \nu \lambda} a_{\mu} \partial_{\nu} a_{\lambda}
$$

Then the effective action for the gauge field $A_{\mu}$ is obtained, in the original formalism, from the path integral

$$
\mathrm{e}^{i \Gamma_{\text {eff }}[A]}=Z[A]=\int \mathcal{D} \Psi^{\dagger} \mathcal{D} \Psi \mathrm{e}^{i S_{\Psi}\left[A_{\mu}, \Psi^{\dagger}, \Psi\right]},
$$

where $S_{\Psi}=\int d t d^{2} x \mathcal{L}_{\Psi}$. On the other hand, after introducing the statistical gauge field, the partition function is

$$
\begin{aligned}
\tilde{Z}[A] & =\int \mathcal{D} a_{\mu} \mathcal{D} \Psi^{\dagger} \mathcal{D} \Psi \mathrm{e}^{i S_{a}\left[a_{\mu}\right]+i S_{\Psi}\left[A_{\mu}+a_{\mu}, \Psi^{\dagger}, \Psi\right]} \\
& =\int \mathcal{D} a_{\mu} \mathrm{e}^{i S_{a}\left[a_{\mu}\right]} Z[A+a]
\end{aligned}
$$

where $S_{a}=\int d t d^{2} x \mathcal{L}_{a}$.

So far all manipulations have been exact. For $\theta=2 k \pi$, with integral $k$, the theory described by (52) is identical to that with no statistical gauge field at all, i.e. $\tilde{Z}[A]=$ $\mathcal{N} Z[A]$, where $\mathcal{N}$ is an irrelevant constant that will be ignored in the sequel. 


\section{APPENDIX B The Relativistic Trick}

We now follow Kivelson, Lee and Zhang 12 and integrate out the matter fields. This cannot be done exactly, of course, but on general grounds one expects the effective action to be invariant under local gauge transformations. Firstly ignore the statistical gauge field and consider the partition function (51). Naïve power counting arguments then imply that the most relevant terms, at least in the infra-red limit, are

$$
\begin{aligned}
\Gamma_{e f f}[A]= & \\
\int d^{3} x d^{3} & x^{\prime}\left\{\left(\frac{1}{2}\right) F_{i 0}(x) \pi_{1}\left(x-x^{\prime}\right) F_{i 0}\left(x^{\prime}\right)\right. \\
& -\left(\frac{1}{2}\right) F_{12}(x) \pi_{2}\left(x-x^{\prime}\right) F_{12}\left(x^{\prime}\right) \\
& \left.-\frac{1}{2} \epsilon^{\mu \nu \lambda} A_{\mu}(x) \pi_{3}\left(x-x^{\prime}\right) \partial_{\nu} A_{\lambda}\left(x^{\prime}\right)\right\}
\end{aligned}
$$

where $F_{i 0}=E_{i}, i=1,2$, is the electric field and $F_{12}=B$ the magnetic field (the measure here $d^{3} x$ is a shorthand for $d t d^{2} x$ and Greek indices, $\mu, \nu, \lambda$ take three values, 0,1 and 2). Note the presence of the non-local form factors $\pi_{1}, \pi_{2}$ and $\pi_{3}$, where the argument $x-x^{\prime}$ includes time as well as space.

There is an implicit assumption here, that the terms that are most relevant by naïve power counting are also the most relevant in the full theory, at least in the infrared limit. This is a very strong assumption, as naïve power counting gives the most relevant operators of the free field theory and we have here a strongly interacting theory. One of the underlying assumptions of Kivelson, Lee and Zhang's argument is therefore that any anomalous dimensions in the strongly interacting theory do not change the conclusions of naïve power counting. In fact the form (53) is rather more general than that - it will be argued below that this is the most general possible form in a momentum expansion, independently of any power counting arguments, provided one allows the polarizations to depend on the magnitudes of the external fields, $\mathbf{E}^{2}$ and $\mathbf{B}^{2}$.

The mathematical manipulations involving equation (53) can be simplified by writing it in relativistic form using the following trick. We introduce a "metric" on 3-dimensional space-time defined by

$$
g_{\mu \nu}\left(x-x^{\prime}\right)=\left(\begin{array}{ccc}
-1 & 0 & 0 \\
0 & \frac{\pi_{1}}{\pi_{2}} & 0 \\
0 & 0 & \frac{\pi_{1}}{\pi_{2}}
\end{array}\right)
$$

and write

$$
\begin{aligned}
\Gamma_{e f f}[A]= & \\
\int d^{3} x d^{3} x^{\prime} & \left\{-\frac{1}{4} \pi_{1}\left(x-x^{\prime}\right) \sqrt{-\operatorname{det}(g)} g^{\mu \rho} g^{\nu \tau} F_{\mu \nu}(x) F_{\rho \tau}\left(x^{\prime}\right)\right. \\
& \left.-\frac{1}{2} \epsilon^{\mu \nu \lambda} A_{\mu}(x) \pi_{3}\left(x-x^{\prime}\right) \partial_{\nu} A_{\lambda}\left(x^{\prime}\right)\right\},
\end{aligned}
$$

where all metric components are functions of $x-x^{\prime}$. Note that the metric does not appear in the Chern-Simons term at all - as is well known it is independent of the metric.

The calculation can be simplified by setting $\pi_{1}=\pi_{2}$ and working in the relativistic formalism with the 3dimensional Minkowski metric, $\eta_{\mu \nu}=\operatorname{diag}(-1,1,1)$, and relativistic action

$$
\begin{aligned}
& \Gamma_{\text {eff }}[A]= \\
& \qquad d^{3} x d^{3} x^{\prime}\left\{-\left(\frac{1}{4}\right) \pi_{1}\left(x-x^{\prime}\right) F^{\mu \nu}(x) F_{\mu \nu}\left(x^{\prime}\right)\right. \\
& \left.\quad-\frac{1}{2} \epsilon^{\mu \nu \lambda} A_{\mu}(x) \pi_{3}\left(x-x^{\prime}\right) \partial_{\nu} A_{\lambda}\left(x^{\prime}\right)\right\},
\end{aligned}
$$

where $F^{\mu \nu}=\eta^{\mu \rho} \eta^{\nu \tau} F_{\rho \tau}$. Provided everything is kept covariant the non-relativistic expressions can be recovered by re-instating the metric (54) at the end. From now on we shall use the simpler relativistic notation of equation (56).

In relativistic notation we can argue that (56) actually encodes higher order terms too. In strong fields one would expect terms such as $F^{\mu}{ }_{\nu} F^{\nu}{ }_{\rho} F^{\rho}{ }_{\lambda} F^{\lambda}{ }_{\mu}$, and other Lorentz invariant powers, to be present in the effective action even in the low momentum regime - though terms like $\partial^{2} F^{\mu \nu} F_{\mu \nu}$ are definitely ignored. In three dimensions terms like $F^{\mu}{ }_{\nu} F^{\nu}{ }_{\rho} F^{\rho}{ }_{\lambda} F^{\lambda}{ }_{\mu}$ can be absorbed into (56) by allowing the polarizations to depend on the Lorentz scalar $F_{\mu \nu} F^{\mu \nu}$. This is because we can always exchange the anti-symmetric tensor $F_{\mu \nu}$ for the vector $\tilde{F}^{\mu}=\epsilon^{\mu \nu \rho} F_{\nu \rho}$ and the only way to make a Lorentz scalar from products of $\tilde{F}^{\mu_{1}} \ldots \tilde{F}^{\mu_{n}}$ is to contract the indices in pairs (and $n$ must be even), so all such terms can simply be incorporated into the statement that $\pi_{1}$ depends analytically on the Lorentz scalar $F^{2}=F^{\mu \nu} F_{\mu \nu}$ and then Taylor expanding $\pi_{1}$ in powers of $F^{2}$. 35 An exactly similar statement applies to $\pi_{3}$. We shall continue with the form (56), bearing in mind that when the external fields are strong the polarizations may depend on them explicitly.

It will be more convenient to work in momentum space where (56) reads

$$
\begin{aligned}
\Gamma_{e f f}[A]=\int d^{3} \bar{p}\{ & -\left(\frac{1}{4}\right) \pi_{1}(p) F^{\mu \nu}(-p) F_{\mu \nu}(p) \\
& \left.-\frac{i}{2 \hbar} \epsilon^{\mu \nu \lambda} A_{\mu}(-p) \pi_{3}(p) p_{\nu} A_{\lambda}(p)\right\},
\end{aligned}
$$

with $d^{3} \bar{p}=\frac{d^{3} p}{\hbar^{3}}$

\footnotetext{
${ }^{\S}$ In general this would necessitate the introduction of multipoint interactions with $n$ points, $x_{1}, \ldots, x_{n}$, but in the long wavelength limit two point interactions suffice to extract conductivities.
} 
A more compact way of writing $(57)$ is

$$
\begin{aligned}
\Gamma_{e f f}[A]=-\int d^{3} \bar{p}\{ & \frac{1}{2} \Pi_{1}(p) A_{\mu}(-p) \Lambda^{\mu \nu} A_{\nu}(p) \\
+ & \left.\frac{1}{2} \Pi_{3}(p) J^{\mu \nu} A_{\mu}(-p) A_{\nu}(p)\right\},
\end{aligned}
$$

where $\Lambda_{\nu}^{\mu}=\delta_{\nu}^{\mu}-\frac{p^{\mu} p_{\nu}}{p^{2}}, J^{\mu \nu}=i \epsilon^{\mu \lambda \nu} p_{\lambda} / \sqrt{p^{2}}, \Pi_{1}=$ $\pi_{1} p^{2} / \hbar^{2}$ and $\Pi_{3}=\pi_{3} \sqrt{p^{2}} / \hbar$. This form is useful, since $\Lambda^{\mu}{ }_{\alpha} \Lambda^{\alpha}{ }_{\nu}=\Lambda^{\mu}{ }_{\nu}, \Lambda^{\mu}{ }_{\alpha} J^{\alpha \nu}=J^{\mu \alpha} \Lambda^{\alpha}{ }_{\nu}=J^{\mu \nu}$ and $J^{\mu \alpha} J_{\alpha \nu}=-\Lambda^{\mu}{ }_{\nu}$, which makes Gaussian integrals particularly simple. Note that $J$ is a Hermitian matrix for space-like momenta, but anti-Hermitian for time-like momenta - however $\sqrt{p^{2}} J$ is always Hermitian, which is all that is necessary to ensure that the momentum space expression is always Hermitian.

It is stressed that (58) is the most general form of the effective action in the long-wavelength limit, even for strong fields, provided $\Pi_{1}$ and $\Pi_{3}$ are allowed to depend on $|\mathbf{E}|^{2}$ and $|\mathbf{B}|^{2}$.

\section{APPENDIX C The Duality Prescription}

The purpose of this appendix is to derive the duality transformation from eq. (2) to eq. (4), which we do following the general duality prescription [30]. Our starting point is an extended Lagrangian which is obtained by coupling $\phi$ to a new gauge field, $\mathcal{A}_{\mu}$, which is constrained to be pure gauge:

$$
\begin{aligned}
\mathcal{L}_{\text {ext }}= & -\frac{\pi}{2 \theta} \epsilon^{\mu \lambda \nu} a_{\mu} \partial_{\lambda} a_{\nu}+\mathcal{L}_{p}(\xi, a+A) \\
& -\frac{\kappa}{2}\left[\partial_{\mu} \phi-q_{\phi}\left(a_{\mu}+A_{\mu}+\mathcal{A}_{\mu}\right)\right]^{2} \\
& +\epsilon^{\mu \nu \lambda} b_{\mu} \partial_{\nu} \mathcal{A}_{\lambda}+\cdots,
\end{aligned}
$$

where the field $b_{\mu}$ is a Lagrange-multiplier field which is introduced to enforce the vanishing of the field strength, $\partial_{\mu} \mathcal{A}_{\nu}-\partial_{\nu} \mathcal{A}_{\mu}$

That this extended action is precisely equivalent to the original action may be seen by first performing the integration over $b_{\mu}$, which produces a functional delta function which enforces the constraint $\epsilon^{\mu \nu \lambda} \partial_{\nu} \mathcal{A}_{\lambda}=0$. This, together with the gauge fixing condition, implies that the integration over $\mathcal{A}_{\mu}$ is equivalent to setting $\mathcal{A}_{\mu}=$ 0 everywhere in the path integral, which reduces eq. (59) to (2), as claimed.

The dual version of the lagrangian is found by instead performing the functional integrals in a different order, integrating out $\phi$ and $\mathcal{A}_{\mu}$ and leaving $b_{\mu}$ as the dual variable. Care must be taken when performing these integrals to properly handle the vortex boundary condition which is satisfied by $\phi$, namely:

$$
\phi(\vartheta+2 \pi)=\phi(\vartheta)+2 \pi q_{\phi} \sum_{a} N_{a}
$$

where $N_{a}$ are integers labelling the vorticity of each vortex, and $\vartheta$ is the angular polar coordinate taken at spatial infinity, a long distance away from the vortex positions.

To integrate over $\phi$ it is convenient to write $\phi=\omega+\varphi$, where $\omega$ is a particular configuration having the same boundary condition as does $\phi$, so $\varphi$ is simply periodic: $\varphi(\vartheta+2 \pi)=\varphi(\vartheta)$. For $\omega$ we choose

$$
\omega(x)=\frac{2 \pi}{q_{\phi}} \sum_{a} N_{a} \arctan \left(\frac{x^{1}-y_{a}^{1}}{x^{2}-y_{a}^{2}}\right),
$$

where $y_{a}^{i}, i=1,2$ are the coordinates of the positions of each vortex, with the index $i$ labelling the two space directions.

Notice that the gauge potential defined by $v_{\mu}=\partial_{\mu} \omega$, has vanishing field strength, except at the positions of the vortices, where it has $\delta$-function singularities, so:

$$
\epsilon^{\mu \nu \lambda} b_{\mu} \partial_{\nu} v_{\lambda}=-\frac{2 \pi}{q_{\phi}} \sum_{a} N_{a} y_{a}^{\mu}(t) b_{\mu} \delta\left[x-y_{a}(t)\right] .
$$

This term appears in the dual lagrangian, and provides the minimal coupling of the vortex positions to the potential $b_{\mu}$.

With these definitions, the integrations over $\varphi$ and $\mathcal{A}_{\mu}$ are straightforward. It is simplest to choose $\varphi=0$ as a gauge condition, and then directly perform the unconstrained Gaussian integral over $\mathcal{A}_{\mu}$. The result is eq. (4), without the vortex kinetic term.

\section{APPENDIX D \\ Particle-Vortex Duality and Landau Level Addition}

In this appendix we derive the duality transformation between pseudo-particles and vortices, but within a second-quantized path-integral framework. (See also ref. 63. for a more detailed discussion of particle/vortex physics within the abelian Higgs model supplemented by a Chern Simons term.) The treatment here is based on [62], except that we use a relativistic notation since this makes the manipulations simpler and, as shown in Appendix $\mathrm{B}$, the non-relativistic form is easily recovered from the relativistic form.

We start from the action for a complex scalar field $\Phi$, with charge $e$, coupled to an external electromagnetic field $A_{\mu}$ with a statistical gauge field $a_{\mu}$

$$
S[\Phi, A, a]=-\int d^{3} x\left[\frac{\pi e^{2}}{2 \theta h} \epsilon^{\mu \nu \rho} a_{\mu} \partial_{\nu} a_{\rho}\right]+S_{m}[\Phi, \tilde{a}]
$$

with

$$
\begin{aligned}
& S_{m}[\Phi, \tilde{a}]= \\
& -\frac{1}{2} \int d^{3} x\left[\left(i \hbar \partial_{\mu}-e \tilde{a}_{\mu}\right) \Phi\right]^{\dagger}\left[\left(i \hbar \partial^{\mu}-e \tilde{a}^{\mu}\right) \Phi\right]+S_{i n t}\left[|\Phi|^{2}\right]
\end{aligned}
$$


where $\tilde{a}=A+a$, and $S_{\text {int }}$ is an interaction term, possibly including a mass term.

\section{- The Flux Attachment Transformation:}

The effective action involving the statistical gauge field should reproduce the same physics as (58), if $\theta=2 k \pi$. To examine this further we first observe that the statistical gauge field $a$ only ever appears in the action (64) in the combination $A+a$, so define $\tilde{a}=\eta A+a$, (at the moment $\eta=1$, but it is introduced here for later convenience). Now integrating the matter fields out of (52) gives rise to an effective action for $A_{\mu}$ and $a_{\mu}$ of the form

$$
\begin{aligned}
\Gamma_{\text {eff }}^{(a)}[A, a]= & -\int d^{3} \bar{p}\left\{\frac{1}{2} \Pi_{1}(p) \tilde{a}_{\mu}(-p) \Lambda^{\mu \nu} \tilde{a}_{\nu}(p)\right. \\
& \left.+\frac{1}{2} \Pi_{3}(p) J^{\mu \nu} \tilde{a}_{\mu}(-p) \tilde{a}_{\nu}(p)\right\} \\
& -\left(\frac{e^{2} \pi}{2 h \theta}\right) \int d^{3} \bar{p} \frac{\sqrt{p^{2}}}{\hbar} J^{\mu \nu} a_{\mu}(-p) a_{\nu}(p) .
\end{aligned}
$$

Even though the matter integrations cannot be done explicitly, the construction ensures that the form factors $\Pi_{1}$ and $\Pi_{3}$ appearing in equation (65) must be identical to those appearing in (58). They will be functions of the field strengths for $\tilde{a}_{\mu}$ in general but, provided $\tilde{a}_{\mu}$ is small (i.e. provided $a_{\mu}$ almost exactly cancels the external field $\eta A_{\mu}$ ), they can be evaluated at $\tilde{a}_{\mu}=0$.

Now equation (65) should describe exactly the same physics as (58), after $a$ is integrated out, and this is what gives rise to the flux attachment transformation, as we now describe.

Integrating the statistical gauge field out of $(65)$ is easily achieved when $\tilde{a}_{\mu}$ is set to zero in $\Pi_{1}$ and $\Pi_{3}$, as it is then quadratic in $a_{\mu}$. The resulting effective action for $A_{\mu}$ will have the same form as (58) with $\Pi_{1}$ and $\Pi_{3}$ evaluated at $A_{\mu}=0$, but with different form factors, which we shall denote by $\tilde{\Pi}_{1}$ and $\tilde{\Pi}_{3}$.

A subtlety in the integration is that in the quadratic from $a_{\mu} M^{\mu \nu} a_{\nu}$ the matrix $M^{\mu \nu}$ is not invertible as it has a zero eigenvalue. However it is only really necessary to find a matrix $\mathcal{M}$ such that, in relativistic formalism in momentum space, $\mathcal{M}_{\mu \nu} M^{\nu \rho}=\delta_{\mu}{ }^{\rho}-\frac{p_{\mu} p^{\rho}}{p^{2}}$ in order to integrate out $a$. The result is 12 ]

$$
\begin{aligned}
\tilde{\Gamma}_{e f f}[A]=-\int d^{3} \bar{p} & \left\{\frac{1}{2} \tilde{\Pi}_{1}(p) A_{\mu}(-p) \Lambda^{\mu \nu} A_{\nu}(p)\right. \\
& \left.+\frac{1}{2} \tilde{\Pi}_{3}(p) J^{\mu \nu} A_{\mu}(-p) A_{\nu}(p)\right\},
\end{aligned}
$$

where

$$
\begin{aligned}
& \tilde{\Pi}_{1}=\left(\frac{e^{2} \eta \pi}{h \theta}\right)^{2} \frac{p^{2} \Pi_{1}}{\hbar^{2} D} \\
& \tilde{\Pi}_{3}=\frac{\sqrt{p^{2}}}{\hbar}\left(\frac{e^{2} \eta^{2} \pi}{h \theta}\right)
\end{aligned}
$$

$$
-\left(\frac{p^{2}}{\hbar^{2}}\right)\left(\frac{e^{2} \eta \pi}{h \theta}\right)^{2}\left(\frac{\Pi_{3}+\frac{\sqrt{p^{2}}}{\hbar}\left(\frac{e^{2} \pi}{h \theta}\right)}{D}\right),
$$

with

$$
\begin{aligned}
D & =\Pi_{1}^{2}+\left[\Pi_{3}+\frac{\sqrt{p^{2}}}{\hbar}\left(\frac{e^{2} \pi}{h \theta}\right)\right]^{2} \\
& =\frac{p^{2}}{\hbar}\left[\frac{p^{2}}{\hbar} \pi_{1}^{2}+\left(\pi_{3}+\frac{e^{2} \pi}{h \theta}\right)^{2}\right] .
\end{aligned}
$$

The non-relativistic transformation is obtained by re-introducing the metric (54) and noting that $p^{2}=$ $g^{\mu \nu} p_{\mu} p_{\nu}=-p_{0}^{2}+\frac{\pi_{2}}{\pi_{1}} \mathbf{p}^{2}$, and then equations (67) become

$$
\begin{aligned}
& \tilde{\pi}_{1}=\left(\frac{e^{2} \eta \pi}{h \theta}\right)^{2} \frac{\pi_{1}}{d} \\
& \tilde{\pi}_{2}=\left(\frac{e^{2} \eta \pi}{h \theta}\right)^{2} \frac{\pi_{2}}{d} \\
& \tilde{\pi}_{3}=\left(\frac{e^{2} \eta^{2} \pi}{h \theta}\right)-\left(\frac{e^{2} \eta \pi}{h \theta}\right)^{2}\left(\frac{\pi_{3}+\left(\frac{e^{2} \pi}{h \theta}\right)}{d}\right),
\end{aligned}
$$

where $d=\left(-\pi_{1}^{2} p_{0}^{2}+\pi_{1} \pi_{2} \mathbf{p}^{2}\right) / \hbar^{2}+\left(\pi_{3}+\frac{e^{2} \pi}{h \theta}\right)^{2}$, and this is the form of the transformation given in [12] (except it is given in Euclidean signature in that reference).

For a Hall conductor the transverse conductivity is related to the polarization tensor $\Pi_{3}$ by

$$
\sigma_{x y}=\pi_{3}=\hbar \Pi_{3} / \sqrt{p^{2}}
$$

while the transverse conductivity involves breaking the 3-momentum up into frequency and spatial momentum $p_{\mu}=(\hbar \omega, \mathbf{p})$ and taking the limit

$$
\begin{aligned}
\sigma_{x x} & =\lim _{\omega \rightarrow 0}\left[\sqrt{p^{2}} \pi_{1} / \hbar\right]_{\mathbf{p}=0}=\lim _{\omega \rightarrow 0}\left[\hbar \Pi_{1} / \sqrt{p^{2}}\right]_{\mathbf{p}=0} \\
& =-i \lim _{\omega \rightarrow 0}\left[\Pi_{1} / \omega\right]_{\mathbf{p}=0} .
\end{aligned}
$$

The transformed conductivities, $\tilde{\sigma}_{x x}$ and $\tilde{\sigma}_{x y}$ are related to the transformed polarization tensors $\tilde{\Pi}_{1}$ and $\tilde{\Pi}_{3}$ in a similar way.

Equation (69) then gives, with $\eta=1, \theta=2 k \pi$ and units in which $e^{2} / h=1$,

$$
\begin{aligned}
& \tilde{\sigma}_{x x}=\frac{\sigma_{x x}}{4 k^{2}\left(\sigma_{x x}^{2}+\sigma_{x y}^{2}\right)-4 k \sigma_{x y}+1} \\
& \tilde{\sigma}_{x y}=\frac{2 k\left(\sigma_{x x}^{2}+\sigma_{x y}^{2}\right)+\sigma_{x y}}{4 k^{2}\left(\sigma_{x x}^{2}+\sigma_{x y}^{2}\right)-4 k \sigma_{x y}+1} .
\end{aligned}
$$

It is stressed here that the above analysis represents a symmetry under certain conditions, as discussed in [12], such as very low temperatures. In the long-wavelength, zero frequency limit, the phase diagram of the quantum Hall effect is symmetric at low temperatures under the 
above transformation. For example the critical exponents at related second order phase transitions should be identical.

It should be remembered that $\sigma_{x x}$ and $\sigma_{x y}$ represent the components of a tensor, it may at first sight seem unnatural to be applying a non-linear map which mixes up the different components of a tensor - what about co-variance of the tensor components? In fact equations (72) are very natural from this point of view. If we define a complex co-ordinate $z:=x+i y$, and it's conjugate $\bar{z}=x-i y$, then the conductivity tensor in these coordinates is reduced to a single quantity, $\sigma:=\sigma_{x y}+$ $i \sigma_{x x}$, with a positive imaginary part, since $\sigma_{x x}>0$. The transformation reduces, in this co-ordinate system, to

$$
\tilde{\sigma}=\eta^{2} \frac{\sigma}{\left(1+\frac{\theta}{\pi} \sigma\right)}
$$

which gives $\tilde{\sigma}=\frac{\sigma}{1+2 k \sigma}$ for $\eta=1$ and $\theta=2 k \pi$. This last form can be obtained by $k$ iterations of the generating transformation

$$
\tilde{\sigma}=\frac{\sigma}{1+2 \sigma}
$$

as is easily checked. Equation (74) is the transformation $S T^{-2} S$ in the text.

\section{- Particle-Vortex Duality:}

We could consider eq. (64) as either: i) a bosonic problem which is transformed from another bosonic problem $(\theta=2 k \pi)$ or ii) a bosonic problem transformed from a fermionic problem $(\theta=(2 k+1) \pi)$ - only in the former case is the statistical gauge field transformation a symmetry.

It will be argued in this section, following [62], that there is a second symmetry in the bosonic case ( $k$ even), $\sigma \rightarrow-1 / \sigma$. This is a $\mathbf{Z}_{2}$ symmetry which maps an insulator $\sigma=0$ to a superconductor $\sigma=i \infty$ and has $\sigma_{x x}=e^{2} / h, \sigma_{x y}=0$ as a fixed point.

We derive the second duality transformation as follows: firstly write 63 in terms of the pseudo-particle paramagnetic current, $j_{\mu}=\frac{i e \hbar}{2}\left[\Phi^{\dagger} \partial_{\mu} \Phi-\left(\partial_{\mu} \Phi\right)^{\dagger} \Phi\right]$, as

$$
\begin{aligned}
S[\Phi, A, a] & =-\int d^{3} x\left[\frac{\pi e^{2}}{2 \theta h} \epsilon^{\mu \nu \rho} a_{\mu} \partial_{\nu} a_{\rho}\right] \\
+\int & d^{3} x\left[-\frac{\hbar^{2}}{2}\left(\partial_{\mu} \Phi^{\dagger}\right)\left(\partial^{\mu} \Phi\right)-\frac{e^{2}}{2}|\Phi|^{2} \tilde{a}^{\mu} \tilde{a}_{\mu}+\tilde{a}^{\mu} j_{\mu}\right] \\
& +S_{\text {int }}\left[|\Phi|^{2}\right] .
\end{aligned}
$$

Alternatively the action (63) can be written in terms of the vortex current by splitting $\Phi$ up into a smooth part and a vortex part as

$$
\Phi(\mathbf{r})=\Phi_{0}(\mathbf{r}) e^{-i \vartheta(\mathbf{r})} v(\mathbf{r})
$$

where $\Phi_{0}(\mathbf{r})$ is real, $\vartheta(\mathbf{r})$ is real, positive and single valued and

$$
v(\mathbf{r})=e^{i \frac{2 \pi}{q_{\phi}} \sum_{a} N_{a} \arctan \left(\frac{x^{1}-y_{a}^{1}}{x^{2}-y_{a}^{2}}\right)},
$$

where $\left(y_{a}^{1}, y_{a}^{2}\right)$ denote the position of the vortex labelled by $a$, which should be summed over in the path integral. In these variables the matter action reads

$$
\begin{aligned}
S_{m}\left[\Phi_{0}, \vartheta, \tilde{a}\right] & =-\frac{1}{2} \int d^{3} x\left[\left(\hbar \partial_{\mu} \Phi_{0}\right)^{2}\right. \\
& \left.+\Phi_{0}^{2}\left(\hbar \partial_{\mu} \vartheta+i \hbar v^{*} \partial_{\mu} v-e \tilde{a}_{\mu}\right)^{2}\right]+S_{i n t}\left[|\Phi|^{2}\right]
\end{aligned}
$$

where indices are understood to be contracted with the Minkowski metric $(-1,+1,+1)$.

We can now perform the integral over $\vartheta$ by introducing an auxiliary vector field, $\lambda_{\mu}=\hbar \partial_{\mu} \vartheta$, and imposing the constraint $\partial_{\mu} \lambda_{\nu}-\partial_{\nu} \lambda_{\mu}=0$ with a Lagrange multiplier field, $\tilde{b}_{\mu}$. So we write

$$
\begin{aligned}
& \int \mathcal{D} \vartheta e^{-\frac{i}{2 \hbar} \int d^{3} x \Phi_{0}^{2}\left(\hbar \partial_{\mu} \vartheta+i \hbar v^{*} \partial_{\mu} v-e \tilde{a}_{\mu}\right)^{2}}= \\
& \int \mathcal{D} \lambda \mathcal{D} b e^{-\frac{i}{2 \hbar} \int d^{3} x \Phi_{0}^{2}\left(\lambda_{\mu}+i \hbar v^{*} \partial_{\mu} v-e \tilde{a}_{\mu}\right)^{2}-\frac{i}{\hbar e} \int d^{3} x \epsilon^{\mu \nu \rho} \tilde{b}_{\mu} \partial_{\nu} \lambda_{\rho}} .
\end{aligned}
$$

Performing the functional integral over $\lambda_{\mu}$ puts (63) in the form

$$
\begin{gathered}
S\left[\Phi_{0}, A, a, b\right]=\int d^{3} x\left[-\frac{\pi}{2 \theta} \frac{e^{2}}{h} \epsilon^{\mu \nu \rho} a_{\mu} \partial_{\nu} a_{\rho}\right. \\
-\epsilon^{\mu \nu \rho} \tilde{a}_{\mu} \partial_{\nu} \tilde{b}_{\rho}+\tilde{j}^{\mu} \tilde{b}_{\mu}-\frac{1}{4 e^{2} \Phi_{0}^{2}} \tilde{f}_{\mu \nu}^{b}\left(\tilde{f}^{b}\right)^{\mu \nu} \\
\left.-\frac{1}{2} \hbar^{2} \partial_{\mu} \Phi_{0} \partial^{\mu} \Phi_{0}\right]+S_{i n t}^{\prime}\left[\Phi_{0}^{2}\right],
\end{gathered}
$$

where $\tilde{f}_{\mu \nu}^{b}=\partial_{\mu} \tilde{b}_{\nu}-\partial_{\nu} \tilde{b}_{\mu}$ is the field strength for $\tilde{b}_{\mu}$ and the vortex current is $\tilde{j}^{\mu}=\frac{i \hbar}{e} \epsilon^{\mu \nu \lambda}\left(\partial_{\nu} v^{*}\right)\left(\partial_{\lambda} v\right)$. The integration over $\lambda$ also induces a functional determinant, $\ln \left(\operatorname{det} \Phi_{0}\right)$ and this has been absorbed into the interaction term for $\Phi_{0}$, as indicated by the prime on $S_{i n t}^{\prime}\left[\Phi_{0}^{2}\right]$.

Now integrate $a_{\mu}$ out of (80). The only terms involving $a$ are

$$
S_{a}=-\int d^{3} x\left[\frac{\pi}{2 \theta} \frac{e^{2}}{h} \epsilon^{\mu \nu \lambda} a_{\mu} \partial_{\nu} a_{\lambda}+\epsilon^{\mu \nu \lambda} a_{\mu} \partial_{\nu} \tilde{b}_{\lambda}\right] .
$$

So integrating out $a$ we get a term

$$
\int d^{3} x\left[\frac{\theta}{2 \pi} \frac{h^{2}}{e} \epsilon^{\mu \nu \lambda} \tilde{b}_{\mu} \partial_{\nu} \tilde{b}_{\lambda}\right]
$$

The action in terms of $\tilde{b}$ is now

$$
\begin{gathered}
S^{(b)}\left[\Phi_{0}, A, \tilde{b}\right]=\int d^{3} x\left[\frac{\theta}{2 \pi} \frac{h}{e^{2}} \epsilon^{\mu \nu \rho} \tilde{b}_{\mu} \partial_{\nu} \tilde{b}_{\rho}\right] \\
+\int d^{3} x\left[-\frac{1}{4 e^{2} \Phi_{0}^{2}} \tilde{f}_{\mu \nu}^{b}\left(\tilde{f}^{b}\right)^{\mu \nu}-\epsilon^{\mu \nu \lambda} A_{\mu} \partial_{\nu} \tilde{b}_{\lambda}+\tilde{b}^{\mu} \tilde{j}_{\mu}\right] \\
-\int d^{3} x\left[\frac{\hbar^{2}}{2} \partial_{\mu} \Phi_{0} \partial^{\mu} \Phi_{0}\right]+S_{i n t}^{\prime}\left[\Phi_{0}^{2}\right] .
\end{gathered}
$$


Now let $\tilde{b}=b+\frac{e^{2}}{h} \frac{\pi}{\theta} A$ and the action becomes

$$
\begin{aligned}
S^{(b)}\left[\Phi_{0}, A, b\right] & =-\int d^{3} x\left[\frac{\pi}{2 \theta} \frac{e^{2}}{h} \epsilon^{\mu \nu \rho} A_{\mu} \partial_{\nu} A_{\rho}\right] \\
& -\int d^{3} x\left[\frac{\pi}{2 \tilde{\theta}} \frac{h}{e^{2}} \epsilon^{\mu \nu \rho} b_{\mu} \partial_{\nu} b_{\rho}\right] \\
& +\int d^{3} x\left[-\frac{1}{4 e^{2} \Phi_{0}^{2}} \tilde{f}_{\mu \nu}^{b}\left(\tilde{f}^{b}\right)^{\mu \nu}+\tilde{b}_{\mu} \tilde{j}^{\mu}\right] \\
& -\int d^{3} x\left[\frac{\hbar^{2}}{2} \partial_{\mu} \Phi_{0} \partial^{\mu} \Phi_{0}\right]+S_{i n t}^{\prime}\left[\Phi_{0}^{2}\right],
\end{aligned}
$$

where $\tilde{\theta}=-\frac{\pi^{2}}{\theta}$.

For comparison (75) reads, writing $\Phi=\Phi_{0} e^{-i \vartheta}$ so that the pseudo-particle para-magnetic current is $j_{\mu}=$ $e \hbar \Phi_{0}^{2} \partial_{\mu} \vartheta$,

$$
\begin{aligned}
S^{(a)}\left[\Phi_{0}, A, a\right]= & -\int d^{3} x\left[\frac{\pi}{2 \theta} \frac{e^{2}}{h} \epsilon^{\mu \nu \rho} a_{\mu} \partial_{\nu} a_{\rho}\right] \\
& +\int d^{3} x\left[-\frac{e^{2} \Phi_{0}^{2}}{2} \tilde{a}_{\mu} \tilde{a}^{\mu}+\tilde{a}^{\mu} j_{\mu}\right] \\
- & \int d^{3} x\left[\frac{\hbar^{2}}{2}\left(\partial_{\mu} \Phi_{0}\right)^{2}+\frac{1}{2 e^{2} \Phi_{0}^{2}} j_{\mu} j^{\mu}\right]+S_{i n t}\left[\Phi_{0}^{2}\right] .
\end{aligned}
$$

The duality symmetry that we seek lies in the symmetry between (84) and (85). One way to see this is to fix the external field $A_{\mu}$ at some background value and treat $\Phi_{0}$ as a classical field. If $\Phi_{0}$ is non-zero constant the action (85) describes a superconductor while (84) describes an insulator - as argued in [1] vortices condense to give an insulator. If the gauge symmetry is not broken $S^{(a)}$ and $S^{(b)}$ describe conductors dual to one another. To examine this situation we analytically continue $\Phi_{0}^{2}$ to complex values and set $e^{2} \Phi_{0}^{2} \approx \frac{e^{2}}{h} \frac{\sqrt{p^{2}}}{\hbar} C=\frac{i e^{2}}{h} \omega C$ where $C$ is dimensionless (this is the leading term in the Fourier expansion of $\Phi_{0}^{2}$, in the long wavelength limit when the gauge symmetry is not broken). So the longitudinal conductivity is (setting $\left.p^{\mu}=(\hbar \omega, \mathbf{0})\right)$

$$
\sigma_{x x}^{a}=\hbar e^{2} \Phi_{0}^{2} / \sqrt{p^{2}}=\lim _{\omega \rightarrow 0}\left(\frac{e^{2} \Phi_{0}^{2}}{i \omega}\right)=C \frac{e^{2}}{h}
$$

(see equation (71) with $\pi_{1}=\hbar^{2} e^{2} \Phi_{0}^{2} / p^{2}$ ). Similarly $S^{(b)}$ describes a conductor with longitudinal conductivity

$$
\sigma_{x x}^{b}=\lim _{\omega \rightarrow 0}\left(\frac{i \omega}{e^{2} \Phi_{0}^{2}}\right)=\frac{1}{C} \frac{h}{e^{2}},
$$

(equation (71) with $\left.\pi_{1}=1 /\left(e \Phi_{0}\right)^{2}\right)$.

For a fixed external field $\Phi_{0}$, and so the conductivities $\sigma_{x x}^{a}$ and $\sigma_{x x}^{b}$, depend on the external field as well as other external parameters, such as the temperature. In general, therefore, it should be possible to find pairs of values for the external parameters, labelled generically by $X$, such that $C(X)=1 / C\left(X^{\prime}\right)$. (this is equivalent to setting $\tilde{\mathbf{V}}=\mathbf{P}$ in section IV). Then the effective actions (84) and (85) are identical except for the extra Chern-Simons term for $A_{\mu}$ in (84).

In particular if we take $\theta=-\pi$ in (84) and $\pi$ in (85) (these two values of $\theta$ are of course indistinguishable in the statistical gauge field transformation) the sole effect of the extra term on the effective action is to shift the transverse conductivity by $\sigma_{x y} \rightarrow \sigma_{x y}+1$ (where we have set $e^{2} / h=1$ ). Of course $\theta= \pm \pi$ means that we started from fermions and transformed to bosons using the statistical gauge field so we can argue that we have derived the $T$ transformation (which corresponds to Landau-Level addition in the first quantised theory) as follows: start with a fermionic system and transform to a bosonic system using $\theta= \pm \pi$, then use the above argument to show that $\sigma \rightarrow \sigma+1$ is a symmetry and then transform back to fermions. This shows that $\sigma \rightarrow \sigma+1$ is a symmetry for the fermionic system (it is not a symmetry for a bosonic system, because $\theta= \pm \pi$ does not keep bosons as bosons). This argument, while plausible, has ignored the currents and the functional integration over $\Phi_{0}$.

A more convincing argument takes the currents, both pseudo-particle and vortex, into account. Pseudo(85)articles and vortices are massive and so should decouple in the long wavelength limit. Just as in the derivation of the flux attachment transformation we can argue that, in the long wavelength limit, integrating out $\Phi_{0}$ from (85) and summing over pseudo-particle currents must lead to an effective action for the external gauge field of the form

$$
\begin{aligned}
& \Gamma_{e f f}^{(a)}[A, a]= \\
&-\int d^{3} x d^{3} x^{\prime}\left\{\left(\frac{1}{4}\right)\left(\tilde{f}^{a}\right)_{\mu \nu}(x) \pi_{1}^{a}\left(x-x^{\prime}\right)\left(\tilde{f}^{a}\right)^{\mu \nu}\left(x^{\prime}\right)\right. \\
&\left.+\frac{1}{2} \epsilon^{\mu \nu \lambda} \tilde{a}_{\mu}(x) \pi_{3}^{a}\left(x-x^{\prime}\right) \partial_{\nu} \tilde{a}_{\lambda}\left(x^{\prime}\right)\right\} \\
& \quad-\frac{e^{2}}{h}\left(\frac{\pi}{2 \theta}\right) \int d^{3} x \epsilon^{\mu \nu \lambda} a_{\mu}(x) \partial_{\nu} a_{\lambda}(x),
\end{aligned}
$$

with $\tilde{f}_{\mu \nu}^{a}=\partial_{\mu} \tilde{a}_{\nu}-\partial_{\nu} \tilde{a}_{\mu}=\partial_{\mu}\left(a_{\nu}+A_{\nu}\right)-\partial_{\nu}\left(a_{\mu}+A_{\mu}\right)$ (we have set $\pi_{2}^{a}=\pi_{1}^{a}$ in the relativistic form - the superscript $a$ indicates that these are polarization tensors associated with the field $a_{\mu}$ ). As before integrating out $\tilde{a}$ now gives an effective action for the external field $A$,

$$
\begin{aligned}
& \tilde{\Gamma}_{e f f}[A]=-\frac{1}{2} \int d^{3} \bar{p}\left\{\frac{p^{2}}{\hbar^{2}} \tilde{\pi}_{1}(p) A_{\mu}(-p) \Lambda^{\mu \nu} A_{\nu}(p)\right. \\
&\left.+\frac{\sqrt{p^{2}}}{\hbar} \tilde{\pi}_{3}(p) J^{\mu \nu} A_{\mu}(-p) A_{\nu}(p)\right\},
\end{aligned}
$$

with polarizations

$$
\begin{aligned}
& \tilde{\pi}_{1}(\omega, \mathbf{p})=\left(\frac{e^{2} \pi}{h \theta}\right)^{2} \frac{\pi_{1}^{a}}{d^{a}} \\
& \tilde{\pi}_{3}(\omega, \mathbf{p})=\frac{e^{2} \pi}{h \theta}-\left(\frac{e^{2} \pi}{h \theta}\right)^{2}\left(\frac{\pi_{3}^{a}+\left(\frac{e^{2} \pi}{h \theta}\right)}{d^{a}}\right),
\end{aligned}
$$


where $d^{a}=\left(\pi_{1}^{a}\right)^{2}\left(-p_{0}^{2}+\mathbf{p}^{2}\right) / \hbar^{2}+\left(\pi_{3}^{a}+\left(\frac{e^{2} \pi}{h \theta}\right)\right)^{2}$. This leads to complex conductivities that are related by

$$
\tilde{\sigma}=\frac{\sigma^{a}}{\left(1+\frac{\theta}{\pi} \sigma^{a}\right)},
$$

with $\sigma^{a}=\lim _{\omega \rightarrow 0}\left(\pi_{3}^{a}-\omega \pi_{1}^{a}\right)$ and $e^{2} / h=1$ (remember $\left.\lim _{\omega \rightarrow 0}\left(\omega \pi_{1}^{a}\right)=-i \sigma_{x x}^{a}\right)$.

Similarly integrating out $\Phi_{0}$ and summing over vortex configurations in (84) must lead, in the long wavelength limit, to

$$
\begin{aligned}
\Gamma_{e f f}^{(b)}[A, b] & = \\
- & \int d^{3} x d^{3} x^{\prime}\left\{\left(\frac{1}{4}\right)\left(\tilde{f}^{b}\right)_{\mu \nu}(x) \pi_{1}^{b}\left(x-x^{\prime}\right)\left(\tilde{f}^{b}\right)^{\mu \nu}\left(x^{\prime}\right)\right. \\
& \left.+\frac{1}{2} \epsilon^{\mu \nu \lambda} \tilde{b}_{\mu}(x) \pi_{3}^{b}\left(x-x^{\prime}\right) \partial_{\nu} \tilde{b}_{\lambda}\left(x^{\prime}\right)\right\} \\
& -\frac{e^{2}}{h} \int d^{3} x \frac{\pi}{2 \theta} \epsilon^{\mu \nu \rho} A_{\mu} \partial_{\nu} A_{\rho} \\
& -\frac{\pi}{2 \tilde{\theta}} \frac{h}{e^{2}} \int d^{3} x \epsilon^{\mu \nu \lambda} b_{\mu}(x) \partial_{\nu} b_{\lambda}(x) .
\end{aligned}
$$

Remember that here $\left(\tilde{f}^{b}\right)_{\mu \nu}=\partial_{\mu}\left(b_{\nu}+\frac{e^{2}}{h} \frac{\pi}{\theta} A_{\nu}\right)-\partial_{\nu}\left(b_{\mu}+\right.$ $\left.\frac{e^{2}}{h} \frac{\pi}{\theta} A_{\mu}\right)$.

Now we can integrate $b$ out of (92) in exactly the same way as $a$ was integrated out of (88) - the only differences are i) the presence of the term involving $-\frac{e^{2}}{h} \frac{\pi}{2 \theta} \epsilon^{\mu \nu \rho} A_{\mu} \partial_{\nu} A_{\rho}$ which subtracts $\frac{e^{2} \pi}{h \theta}$ from the right hand side of $\tilde{\pi}_{3}$ in (90), ii) $\frac{e^{2} \pi}{h \theta}$ is replaced with $\frac{h \pi}{e^{2} \theta}$ with $\tilde{\theta}=-\frac{\pi^{2}}{\theta}$ in (90); iii) we should use $\eta=\frac{e^{2} \pi}{h \theta}$ and iv) the $\pi_{i}^{a}$ are replaced by $\pi_{i}^{b}$, which will in general be different. The resulting effective action is

$$
\begin{aligned}
\tilde{\tilde{\Gamma}}_{e f f}[A]=-\frac{1}{2} \int d^{3} \bar{p} & \left\{\frac{p^{2}}{\hbar^{2}} \tilde{\pi}_{1}(p) A_{\mu}(-p) \Lambda^{\mu \nu} A_{\nu}(p)\right. \\
+ & \left.\frac{\sqrt{p^{2}}}{\hbar} \tilde{\tilde{\pi}}_{3}(p) J^{\mu \nu} A_{\mu}(-p) A_{\nu}(p)\right\},
\end{aligned}
$$

with different polarization transformations to $(90)$ - see equation (69),

$$
\begin{aligned}
\tilde{\tilde{\pi}}_{1}(\omega, \mathbf{p}) & =\left(\frac{\pi}{\theta}\right)^{2}\left(\frac{\pi}{\tilde{\theta}}\right)^{2} \frac{\pi_{1}^{b}}{d^{b}}=\frac{\pi_{1}^{b}}{d^{b}} \\
\tilde{\tilde{\pi}}_{3}(\omega, \mathbf{p}) & =\left(\frac{e^{2} \pi}{h \theta}\right)^{2} \frac{\pi}{\tilde{\theta}}-\left(\frac{\pi}{\theta}\right)^{2}\left(\frac{\pi}{\tilde{\theta}}\right)^{2}\left(\frac{\pi_{3}^{b}+\left(\frac{h \pi}{e^{2} \theta}\right)}{d^{b}}\right)+\frac{e^{2} \pi}{h \theta} \\
& =\frac{\left(\left(\frac{h \theta}{e^{2} \pi}\right)-\pi_{3}^{b}\right)}{d^{b}}
\end{aligned}
$$

where $d^{b}=\left(\pi_{1}^{b}\right)^{2}\left(-p_{0}^{2}+\mathbf{p}^{2}\right) / \hbar^{2}+\left(\pi_{3}^{b}-\left(\frac{h \theta}{e^{2} \pi}\right)\right)^{2}$.

In terms of complex conductivities (94) results in (again with $e^{2} / h=1$ )

$$
\tilde{\tilde{\sigma}}=-\frac{1}{\left(\sigma^{b}-\frac{\theta}{\pi}\right)},
$$

with $\sigma^{b}=\lim _{\omega \rightarrow 0}\left(\pi_{3}^{b}-\omega \pi_{1}^{b}\right)$. If $\theta=2 \pi k$ then this equation represents a symmetry of a bosonic theory, $\sigma \rightarrow-\frac{1}{\sigma-2 k}$ which is equivalent to the modular transformation $\gamma=\left(\begin{array}{cc}0 & 1 \\ -1 & 2 k\end{array}\right)$. The particular case $k=0$ is $\sigma \rightarrow-1 / \sigma$.

As discussed in Appendix B the effective actions (89) and (93) are long-wavelength but not necessarily weak field if the polarisations depend on the background electric and magnetic fields. This means that the conductivities $\sigma^{a}$ and $\sigma^{b}$ will depend on external parameters, generically labelled $X$ before, including the external magnetic field and the temperature, so consider (93) to be the effective action for a perturbation on the external field. Typically it will be possible to find pairs of parameters so that $\sigma^{a}(X)=\sigma^{b}\left(X^{\prime}\right)$. Then eliminating $\sigma^{a}$ and $\sigma^{b}$ from (91) and (95) gives $S(\tilde{\tilde{\sigma}}=-1 / \tilde{\sigma})$ when $\theta=0$. Together with the flux attachment transformation (74) this generates the group $\Gamma_{\theta}$ for bosons. Conjugating with $S T$ as described in the text gives $\Gamma_{0}(2)$ for fermions.

\section{APPENDIX E: The Gaussian Integrals}

In this appendix we perform in detail the Gaussian integrations which lead to our central formula, eq. (15). Our starting point is eq. (蛋) after the integration over the positions of the quasi-particles and vortices has been performed, using eqs. (9) and (10). This starting point may be written:

$$
\begin{gathered}
S_{0}=-\int d^{3} x\left[\frac{\pi}{2 \theta} \epsilon^{\mu \lambda \nu} a_{\mu} \partial_{\lambda} a_{\nu}+\epsilon^{\mu \lambda \nu} b_{\mu} \partial_{\lambda}(a+A)_{\nu}\right] \\
-\frac{1}{2} \int d^{3} x d^{3} y\left[(a+A)_{\mu}(x) P^{\mu \nu}(x-y)(a+A)_{\nu}(y)\right. \\
\left.+b_{\mu}(x) V^{\mu \nu}(x-y) b_{\nu}(y)\right] .
\end{gathered}
$$

Our task is to perform the integrals over $a_{\mu}$ and $b_{\mu}$, and our interest is in the dependence of the result on $A_{\mu}$. Because none of the functional determinants which arise in the integrations depend on $A_{\mu}$, these may be neglected as contributing an $A_{\mu}$-independent additive constant to the EM response function, $\Gamma[A]$.

Performing first the $b_{\mu}$ integration, the saddle-point condition may be written as:

$$
b_{\mu}(x)=-\int d^{3} y \mathcal{V}_{\mu \nu}(x-y) \epsilon^{\nu \alpha \beta} \partial_{\alpha}(a+A)_{\beta}(y),
$$

where the kernel $\mathcal{V}_{\mu \nu}$ satisfies the definition

$$
\int d^{3} y \mathcal{V}_{\mu \lambda}(x-y) V^{\lambda \nu}(y-z)=\Lambda_{\mu}^{\nu}(x-z),
$$

for the projection operator $\Lambda_{\mu}{ }^{\nu}(x-z)=\left(\delta_{\mu}^{\nu}-\partial_{\mu} \partial^{\nu} / \partial^{2}\right) \delta^{3}(x-z)$.

The result for the effective action then becomes: 


$$
\begin{aligned}
S_{1}= & -\int d^{3} x\left[\frac{\pi}{2 \theta} \epsilon^{\mu \lambda \nu} a_{\mu} \partial_{\lambda} a_{\nu}\right] \\
& -\frac{1}{2} \int d^{3} x d^{3} y(a+A)_{\mu}(x) \hat{P}^{\mu \nu}(x-y)(a+A)_{\nu}(y),
\end{aligned}
$$

where:

$$
\hat{P}^{\mu \nu}=P^{\mu \nu}-\epsilon^{\mu \lambda \rho} \partial_{\lambda} \mathcal{V}_{\rho \alpha} \epsilon^{\alpha \beta \nu} \partial_{\beta} .
$$

The construction of kernels like $\mathcal{V}_{\mu \nu}$ is dramatically simplified by working in momentum space, and expanding the kernels in terms of the basis of tensors $\Lambda_{\mu}{ }^{\nu}$ and $J^{\mu \nu}$, as introduced in the text. For instance, if $V^{\mu \nu}(p)=A_{1} \Lambda^{\mu \nu}+A_{2} J^{\mu \nu}$, then $\mathcal{V}_{\mu \nu}(p)=B_{1} \Lambda_{\mu \nu}+B_{2} J_{\mu \nu}$ with

$$
B_{1}=\frac{A_{1}}{A_{1}^{2}+A_{2}^{2}}, \quad B_{2}=-\frac{A_{2}}{A_{1}^{2}+A_{2}^{2}},
$$

which is more compactly written in terms of the complex variables $\mathbf{A}=A_{1}+i A_{2}$ and $\mathbf{B}=B_{1}+i B_{2}$ as: $\mathbf{B}=1 / \mathbf{A}$.

Proceeding in the same vein, the saddle point for the $a_{\mu}$ integration is

$$
a_{\mu}=-\int d^{3} y \mathcal{K}_{\mu \nu} \hat{P}^{\nu \lambda} A_{\lambda}
$$

where $\mathcal{K}_{\mu \lambda} K^{\lambda \nu}=\Lambda_{\mu}{ }^{\nu}$ and $K^{\mu \nu}=\hat{P}^{\mu \nu}+\frac{\pi}{\theta} \epsilon^{\mu \lambda \nu} \partial_{\lambda}$. Using this in the integration over $a_{\mu}$ gives the electromagnetic response function of eq. (11), with the EM response tensor, $\Pi^{\mu \nu}$ given by:

$$
\Pi^{\mu \nu}=\hat{P}^{\mu \nu}-\hat{P}^{\mu \lambda} \mathcal{K}_{\lambda \rho} \hat{P}^{\rho \nu} .
$$

Once expressed in terms of complex variables, as above, this result gives eq. (15) of the text.

[1] M.P.A. Fisher, Phys. Rev. Lett. 65, 923 (1990).

[2] M.P.A. Fisher and D.H. Lee, Phys. Rev. B39, 2756 (1989).

[3] D.H. Lee and M.P.A. Fisher, Int. J. Mod. Phys. B5, 2675-2699 (1991).

[4] D.-H. Lee and C.L. Kane, Phys. Rev. Lett. 64, 1313 (1990).

[5] A. Shapere and F. Wilczek, Nucl. Phys. B320, 669 (1989).

[6] S.-J. Rey and A. Zee, Nucl. Phys. B352, 897-921 (1991).

[7] A.P. Balachandran, L. Chandar and B. Sathiapalan, Nucl. Phys. B443, 465-500 (1995).

[8] M.C. Diamantini, P. Sodano and C.A. Trugenberger, Nucl. Phys. B448, 505-532 (1995).

[9] J.L. Cardy and E. Rabinovici, Nuc. Phys. B205, 1 (1982); J.L. Cardy, Nuc. Phys. B205, 17 (1982).

[10] R.A. Rankin, Modular Forms and Functions, (Cambridge University Press, 1977).
[11] C.A. Lütken and G.G. Ross, Phys. Rev. B45, 11837 (1992); Phys. Rev. B48, 2500 (1993).

[12] D-H. Lee, S. Kivelson and S-C. Zhang, Phys. Lett. 68, 2386 (1992); S. Kivelson, D-H. Lee and S-C. Zhang, Phys. Rev. B46, 2223 (1992).

[13] C.A. Lütken, Nuc. Phys. B396, 670 (1993).

14] B.P. Dolan, J. Phys. A32, L243 (1999) condmat/9805171.

[15] C.P. Burgess, Rim Dib and B.P. Dolan, To appear in Phys. Rev. B cond-mat/9911476.

[16] C.P. Burgess and C.A. Lütken, Nuc. Phys. B500, 367 (1997) (cond-mat/9611118.

17] B.P. Dolan, Nuc. Phys. 460B [FS], 297 (1999) condmat/9809294.

[18] N. Taniguchi, Nonperturbative Renormalization Group Function for Quantum Hall Plateau Transitions Imposed by Global Symmetries (cond-mat/9810334).

[19] C.P. Burgess and C.A. Lütken, Phys. Lett. B451, 365 (1999) (hep-th/9812396).

[20] E. Abrahams, P.W. Anderson, D.C. Licciardello and T.V. Ramakrishnan, Phys. Rev. Lett. 42, 673 (1979).

[21] D.E. Khmel'nitskii, Pis'ma Zh. Eksp. Teor. Fiz 38, 454 (1983) (JETP Lett. 38, 552 (1983)).

[22] A.M.M. Pruisken, Phys. Rev. Lett. 61, 1297 (1988).

[23] B. Huckelstein, Rev. Mod. Phys. 67, 357 (1995); S.L. Sondhi, S.M. Girvin, J.P. Carini and D. Shahar, Rev. Mod. Phys. 69, 315 (1997).

[24] L. Pryadko, Phys. Rev. B56, 6810 (1997).

[25] D. Arovas, J.R. Schrieffer and F. Wilczek, Phys. Rev. Lett. 53, 722 (1984).

[26] For a recent review see, for example, C.P. Burgess, Phys. Rep. C330, 193 (2000) (hep-ph/9808176).

[27] D.H. Lee and M. Fisher, Phys. Rev. Lett. 63, 903 (1989).

[28] D.H. Lee and S.-C. Zhang, Phys. Rev. Lett. 66, 1220 (1991).

[29] S-C. Zhang, Int. J. Mod. Phys. B 6, 25 (1992).

[30] T. Buscher, Phys. Lett. B194 (1987) 59; Phys. Lett. B201, 466 (1988).

[31] C.P. Burgess and F. Quevedo, Nucl. Phys. 421, 373 (1994); Phys. Lett. B329, 457 (1994); C.P. Burgess, C.A. Lütken and F. Quevedo, Phys. Lett. B336, 18 (1994).

[32] J. Hughes, J. Liu and J. Polchinski, Nucl. Phys. B316, 15 (1989)

[33] See for example, E. Fradkin, Field Theories of Condensed Matter Systems, Addison Wesley, 1991.

[34] R. Laughlin, Science 242, 525 (1988).

[35] V. Kalmeyer and R.B. Laughlin, Phys. Rev. Lett. 59, 2095 (1987).

[36] D. Shahar, D.C. Tsui, M. Shayegan, J.E. Cunningham, E. Shimshoni and S.L. Sondhi, Solid State Comm. 102, 817 (1997) (cond-mat/9607127) D. Shahar, D.C. Tsui, M. Shayegan, E. Shimshoni and S.L. Sondhi, Science 274, 589 (1996) (cond-mat/9510113).

[37] N. Seiberg and E. Witten, Nucl.Phys. B426, 19-52 (1994), Erratum: ibid. B430, 485-486 (1994) (hepth/9407087); Nucl. Phys. B431, 484-550 (1994) (hepth/9408099.

[38] M.P.A. Fisher, G. Grinstein and S.M. Girvin, Phys. Rev. Lett. 64, 587 (1990); M.-C. Cha, M.P.A. Fisher, S.M. Girvin, M. Wallin and A.P. Young, Phys. Rev. B44, 6883 (1991). 
[39] See table 1. in 14 for the relation between the experimental results and the modular group's predictions.

[40] D.E. Khmel'nitskii in ref. 22]; A.M.M. Pruisken, in ref. 22]; R.B. Laughlin, Phys. Rev. Lett. 52, 2304 (1984);

[41] H.P. Wei, D.C. Tsui, M.A. Paalanen and A.M.M. Pruisken, Phys. Rev. Lett. 61, 1294 (1988).

[42] L. Engel et.al., Surf. Sci. 229, 13 (1990).

[43] A.M. Dykhne and I.M. Ruzin, Phys. Rev. B50, 2369 (1994); I. Ruzin and S. Feng, Phys. Rev. Lett. 74, 154 (1995).

[44] M. Hilke et al., Euro. Phys. Lett. 46, 775 (1999) cond-mat/9810217)

[45] M. Hilke, D. Shahar, S.H. Song, D.C. Tsui and Y.H. Xie, Phys. Rev. B62, 6940-6943 (2000) cond-mat/9906212.

[46] We thank M. Hilke for conversations on this point.

[47] E. Shimshoni, Critical Resistivity along the Quantum Hall Liquid-Insulator Transition Line, (cond-mat/0005436).

[48] D. Shahar, M. Hilke, C.C. Li, D.C. Tsui, S.L. Sondhi and M. Razeghi, Solid State Comm. 107, 19-23 (1998) (cond-mat/9706045)

[49] P.T. Coleridge, Phys. Rev. B60, 4493 (1999); P.T. Coleridge, Solid State Comm. 112, 241 (1999)

[50] R. Fazio and G. Schön, Phys. Rev. B43, 5307 (1991).

[51] A. Stern, Phys. Rev. B50 10092-10106 (1994) condmat/9403017).

[52] A.A. Odintsov and Yu. V. Nazarov, Phys. Rev. B51 (1995) 1133-1136.

[53] M.Y. Choi, Phys. Rev. B50, (1994) 10088-10091.

[54] M.C. Diamantini, P. Sodano and C.A. Trugenberger, Nucl. Phys. B474, 641-677 (1996).

[55] N.P. Ong, in Physical Properties of High Temperature Superconductors, Vol. II, Ed. D.M. Ginsperg, World Scientific (1990)

[56] S.V. Kravchenko, D. Simonian, M.P. Sarachik, W. Mason and J.E. Furneaux, Phys. Rev. Lett. 77, 4938 (1996).

[57] D. Simonian, S.V. Kravchenko and M.P. Sarachik, Phys. Rev. B55, 13421-13423 (1997) cond-mat/9611147.

[58] C.A. Lütken in 13] and J. Math. Phys. A 26, L811-L817 (1993).

[59] Y. Georgelin and J-C. Wallet, Phys. Lett. A224, 303308 (1997); Y. Georgelin, T. Masson and J-C. Wallet, J. Phys. A30 5065-5075 (1997); Y. Georgelin, T. Masson and J-C. Wallet, J. Phys. A33, 39-55 (2000) condmat/9906193).

[60] B.P. Dolan, Duality in the Quantum Hall Effect - the Role of Electron Spin, To appear in Phys. Rev. B (cond-mat/0002228).

[61] S.M. Girvin and A.H. MacDonald, Phys. Rev. Lett. 58, 1252 (1987).

[62] A. Karlhede, S.A. Kivelson, and S. Sondhi, Quantum Hall Effect: The Article, in Correlated Electron Systems ed. by V. J. Emery, (World Scientific, Singapore, 1993).

[63] P.W. Irwin and M.B. Paranjape, preprint UDEM-GPPTH-99-63 (hep-th/9906021). 